\title{
Zur Geschichte der ungarischen Rezeption Albrecht von Hallers • Erster Teil
}

\author{
Miklós Latzkovits*
}

\begin{abstract}
Szegedi Tudományegyetem Bölcsészet- és Társadalomtudományi Kar, Egyetem utca 2., 6722 Szeged, Hungary
\end{abstract}

\section{ORIGINAL RESEARCH PAPER}

Received: March 29, 2020 • Accepted: October 18, 2020

Published online: May 13, 2021

(c) 2020 The Author(s)

\section{ABSTRACT}

Der Aufsatz beschäftigt sich mit der Rezeptionsgeschichte der Gedichte Albrecht von Hallers im Ungarn des 18. Jahrhunderts. Als Grundlage für die Untersuchung dienen die Haller-Zitate in zeitgenössischen Stammbucheinträgen. Mit dieser Frage setzten sich zwar sowohl deutsche als auch ungarische Forscher schon früher auseinander, jedoch, wie im vorliegenden Aufsatz behauptet wird, unter Anwendung einer gewissermaßen falschen Methode. Es wurde nämlich in diesen früheren Arbeiten nicht näher darauf eingegangen, ob das Zitat gegebenenfalls einer sekundären Quelle entnommen wurde. Dieser Ansatz führte jedoch zu falschen Schlussfolgerungen. Vorliegender Aufsatz versucht, die aus sekundären Quellen stammenden Zitate von jenen aus dem „Original“ zu unterscheiden. Es wird festgestellt, dass die in Stammbucheinträgen im 18. Jahrhundert zu lesenden HallerZitate überwiegend aus nachweislich sekundären Quellen herzuleiten sind. Je „,berühmter" ein Autor war - diesen Eindruck gewinnt man anhand des Korpus -, umso mehr zitieren ihn die Zeitgenossen aus indirekten Quellen.

\section{KEYWORDS}

Albrecht von Haller, Rezeption im 18. Jahrhundert, ungarische Rezeption, Wirkungsgeschichte, Stammbücher, Zitate

\footnotetext{
*Corresponding author. E-mail: latzkovits@t-online.hu
} 


\section{PROVISORISCHE STATISTIKEN}

In der vorliegenden Arbeit möchte ich die ungarische Rezeption Albrecht von Hallers aus einer besonderen Hinsicht untersuchen. ${ }^{1}$ Ich interessiere mich dafür, wie die Texte des schweizerischen Dichters in Stammbucheinträgen des 18. Jahrhunderts auftauchen. Die zu analysierenden Einträge stammen meist aus Alben ungarischer Besitzer, ich habe aber auch einige Autographe ungarischer Einträger in Alben von ausländischen Besitzern in Betracht gezogen. Die Grundlagen meiner Bemerkungen bilden die Daten, die während der Erstellung der Datenbank Inscriptiones Alborum Amicorum (IAA) aufgearbeitet wurden. ${ }^{2}$ Derzeit kann auf 12,500 Einträge in der IAA zugegriffen werden. ${ }^{3}$ 2,700 enthalten deutschsprachige Textteile. Der älteste ist im Jahr 1553 entstanden, ${ }^{4}$ die meisten der erwähnten Einträge sind aber im 18. Jahrhundert in die untersuchten Alben geschrieben worden (ca. 2,300 Autographe). In insgesamt 53 Fällen wurden Haller-Zitate registriert: Diese bilden die Grundlage der vorliegenden Arbeit.

Als eine Vergleichsbasis, die aus fachlicher Hinsicht wichtig ist, kann ich auf die beinahe 100 Seiten lange Quellenpublikation bzw. den Aufsatz von Klára Berzeviczy und Péter Lökös Bezug nehmen, ${ }^{5}$ die meinem Thema ziemlich ähnlich sind. Als diese beiden Wissenschaftler nach deutschen Gedichten geforscht haben, die in Stammbüchern im 18. Jahrhundert zitiert wurden, wurden insgesamt 21 Alben untersucht, die im Bestand der Ungarischen Nationalbibliothek [Országos Széchényi Könyvtár, Budapest] zu finden sind. Drei dieser 21 Stammbücher haben überhaupt keinen Hungarica-Bezug - außer der Tatsache, dass sie heute in der OSZK aufbewahrt sind. Unter ihren Besitzern und Einträgern gibt es niemanden aus Ungarn; sie wurden aber ebenfalls untersucht, um eine Art Vergleichbarkeit schaffen zu können. ${ }^{6}$ Die Besitzer von 18 Alben waren tatsächlich Hungari, obwohl dies in zwei Fällen nur eine Annahme der Autoren war. ${ }^{7}$ Diese Annahmen waren zwar korrekt, ich würde dennoch hinzufügen, dass das

\footnotetext{
${ }^{1}$ Der Aufsatz in ungarischer Sprache: LATzKoviTs Miklós, „Albrecht von Haller magyarországi recepciójának történetéhez: Haller és a nők“, Irodalomtörténeti Közlemények 122 (2018): 317-355.

${ }^{2}$ Inscriptiones Alborum Amicorum, 2003-2017, doi:10.14232/iaa, http://iaa.bibl.u-szeged.hu. In dieser kontinuierlich zu erweiternden Datenbank veröffentlichen wir Albumeinträge der Art Hungarica aus dem 16.-18. Jahrhundert.

${ }^{3}$ Zuletzt gesehen am 10. Juli 2017.

${ }^{4}$ IAA, 12,117. Dieser Eintrag stammt von Christoph Mandel. Soweit ich weiß, wird zurzeit sein Autograph als der älteste Eintrag betrachtet, der von einem Ungarn stammt. Über Mandel siehe: Wix Györgyné, Régi magyarországi szerzők (RMSz) I. A kezdetektöl 1700-ig [Alte ungarische Autoren I. Von den Anfängen bis 1700] (Budapest, Országos Széchényi Könyvtár, 2008), 513.

${ }^{5}$ Klára BERZEVICZY und Péter LŐKÖs, „Zitate deutscher Dichter des 18. Jahrhunderts in Stammbüchern der Ungarischen Széchényi-Nationalbibliothek: Ein Beitrag zur zeitgenössischen Rezeption der deutschen Literatur des 18. Jahrhunderts in Ungarn“ in „Ars longa, vita academica brevis“: Studien zur Stammbuchpraxis des 16.-18. Jahrhunderts, Herausgegeben von Klára BERZEVICZY und Péter LŐKÖs unter Mitarbeit von Zsófia HORNYÁK (Budapest, Országos Széchényi Könyvtár, 2009), 109-181.

${ }^{6}$ OSZK, Oct. Lat. 116., Oct. Lat. 109., Oct. Lat. 624. Vgl. BERZEVICZY-LőKÖs, „Zitate deutscher. ..“, 133.

${ }^{7}$ OSZK, Oct. Germ. 249., Oct. Lat. 128., Oct. Lat. 457., Oct. Lat. 776., Duod. Hung. 166., Oct. Hung. 619. III., Oct. Lat. 110., Oct. Lat. 117., Oct. Lat. 467., Oct. Lat. 630., Oct. Lat. 718., Oct. Lat. 850., Oct Lat. 1168., Oct. Lat. 1222., Oct. Lat. 1251., Duod. Hung. 177. Die zwei als problematisch erwähnten Alben: OSZK, Duod. Lat. 118. (Joseph Freysmuth) und Oct. Germ. 250. (M. Fistrovits).
} 
Stammbuch von Joseph Freysmuth nicht anonym und auch nicht das Album eines gewissen „Herrn Kriebel“ ist. ${ }^{8}$ Der Nachname Freysmuth taucht (unter anderen) im Eintrag von Johann Karl Volborth, einem Studenten in Göttingen, auf, ${ }^{9}$ sein Vorname in der Leipziger und Göttinger Matrikel. ${ }^{10}$ Über das andere, ebenfalls als problematisch bezeichnete Album kann auch festgestellt werden, dass es nicht das Stammbuch eines „Ehepaars“ ist, sondern das eines jungen Mannes namens M. Fistrovits, dessen Nachname in diesem Fall (zum Beispiel) dem Eintrag seiner Schwester, ${ }^{11}$ der Anfangsbuchstabe seines Vornamens wiederum dem Monogramm auf dem Band $\mathrm{zu}$ entnehmen ist. ${ }^{12}$

Ein offensichtlich ernsteres Problem hinsichtlich der Untersuchung von Berzeviczy und Lőkös ist, dass sie lediglich 18 Alben durchgesehen haben, obwohl in der OSZK 42 weitere Stammbücher aufbewahrt sind, deren Besitzer mit Sicherheit aus Ungarn stammen und deren Einträge im 18. Jahrhundert entstanden sind. ${ }^{13}$ Es ist in der Tat nicht ersichtlich, warum nur diese 18 Alben unter die Lupe genommen und warum die weiteren 42 von der Untersuchung ausgeschlossen wurden. ${ }^{14}$ Ein weiteres Problem ist, dass die gereimten Texte in den einzelnen Stammbüchern in den meisten Fällen - natürlich nicht immer - nur dann identifiziert wurden, wenn die einstigen Einträger selbst angegeben haben, von wem das jeweilige Gedicht stammt. Die Möglichkeiten der Autoren waren offensichtlich durch die CD-ROMs begrenzt, auf die sie Bezug nehmen, bzw. waren sie durch die noch etwas eingeschränkten Möglichkeiten der Recherche im Internet festgelegt. Die Letzteren wurden in den vergangenen zehn Jahren stark erweitert. Vermutlich dadurch erklärt sich auch der Unterschied, dass Berzeviczy und Lőkös zum Beispiel von den 25 Gedichten, die von Samuel Coellnberger ins Peregrinationsalbum

${ }^{8}$ JóNÁCsIK, László: „Miszellen aus der Stammbuchforschung in der Ungarischen Széchényi-Nationalbibliothek Budapest (OSzK)“ in Mitteleuropäischer Kulturraum: Völker und religiöse Gruppen des Königreichs Ungarn in der deutschsprachigen Literatur und Presse, Klára BERZEviCzy - László jóNÁcsiK - Péter LÖKÖs (Hg.), 93-126. (Berlin: Frank \& Timme, 2015), 119. Die Ansprache „Kriebel“ auf Seite 111r des Albums verweist übrigens auf den Eintrag auf der darauffolgenden Seite 111v, der von Samuel Kriebel stammt, der also nicht der Besitzer, sondern auch ein Einträger des Stammbuchs ist. Vgl. IAA, 5,524.

${ }^{9}$ Volborth bezeichnet in der Dedikation den Namen des Stammbuchbesitzers: „mi amicissime Freysmuthi“. Vgl. IAA, 8, 409.

${ }^{10}$ TAR Attila, Magyarországi diákok németországi egyetemeken és fóiskolákon 1694-1789 [Ungarische Studenten an deutschen Universitäten und Hochschulen 1694-1789]. Magyarországi diákok egyetemjárása az újkorban 11. [Auslandsstudien ungarischer Studenten in der frühen Neuzeit 11] (Budapest: Eötvös Loránd Tudományegyetem Levéltára, 2004), 634, 2,784.

${ }^{11}$ „Dieses schrieb zum steten andenken deine Wahre $\mathrm{u}(\mathrm{nd})$ Einzige Schwester, als Ihren Einzigen Lieben Bruder.“ Die Unterschrift: „An(n)a Rosina Friedin gebohrne Fiestrowitschin“. Vgl. IAA, 12,218. Die Frage wird übrigens dadurch endgültig entschieden, dass ein Einträger den Besitzer des Albums mit der Formel „mein Lieber Fistrovits“ anspricht. Vgl. IAA, 12,228.

${ }^{12}$ Das Monogramm auf dem Band: M. F. Der zweite Buchstabe bezeichnet natürlich den Nachnamen (Fistrovits).

${ }^{13}$ Duod. Germ. 58., Duod. Germ. 70., Duod. Germ. 71., Oct. Germ. 399., Oct. Hung. 619. II., Oct. Hung. 627., Oct. Hung. 1061., Oct. Lat. 123., Oct. Lat. 124., Oct. Lat. 127., Oct. Lat. 130., Oct. Lat. 131., Oct. Lat. 452., Oct. Lat. 454., Oct. Lat. 455., Oct. Lat. 460., Oct. Lat. 1255., Oct. Lat. 1256., Duod. Germ. 45., Duod. Lat. 152., Oct. Germ. 446., Oct. Germ. 555., Oct. Germ. 596., Oct. Hung. 1940., Oct. Lat. 86., Oct. Lat. 111., Oct. Lat. 112., Oct. Lat. 113., Oct. Lat. 118., Oct. Lat. 121., Oct. Lat. 122., Oct. Lat. 134., Oct. Lat. 389., Oct. Lat. 458., Oct. Lat. 605., Oct. Lat. 687., Oct. Lat. 777., Oct. Lat. 850., Oct. Lat. 1,221., Oct. Lat. 1,236., Oct. Lat. 1,247., Quart. Lat. 547. 
kopiert wurden, lediglich drei identifizieren konnten (alle drei stammen von Albrecht von Haller und die Einträger geben dies auch jedes Mal an), ${ }^{15}$ wogegen die Quelle der einzelnen Gedichte heute auch noch in weiteren zehn Fällen bestimmt werden kann. Die im Album von Coellnberger zitierten deutschen Dichter sind also die Folgenden: Haller in weiteren zwei (also insgesamt fünf) Fällen. ${ }^{16}$ Wir haben ferner ein Zitat von Johann Christian Günther und eines von Johann Ludwig Huber, ${ }^{17}$ in jeweils zwei Fällen von Johann Friedrich von Cronegk, Johann Jacob Dusch und Johann Peter Uz. ${ }^{18}$ Die Einträger haben in den meisten Fällen nicht den Namen des zitierten Dichters erwähnt, in zwei Fällen ist dies jedoch geschehen. Joseph Friedrich Matolay, ein junger Mann, der in Erlangen studiert hat und aus Wien stammte, kennzeichnet zum Beispiel eindeutig, wen er zitiert (,Dusch“), ${ }^{19}$ und Ludwig Achatius Mohr tut dasselbe

\footnotetext{
${ }^{14}$ Klára Berzeviczy berichtet als Ergänzung in einem Aufsatz von 2017 von der Untersuchung sieben weiterer Stammbücher, unter denen drei (die eigentlich schon im Aufsatz von 2009 analysiert, hier aber mit größerer Wirksamkeit untersucht wurden) im Bestand der OSZK zu finden sind. Zwei von diesen sieben Stammbüchern sind zurzeit nicht im IAA aufgearbeitet, nämlich die Alben von Dániel Cornides (OSZK, Oct. Germ. 249.) und von Gergely Berzeviczy. Das Letztere ist derzeit allerdings verschollen, die zum Verkauf angebotene Handschrift wurde nämlich 2009 aus einer Vitrine der Frankfurter Buchmesse gestohlen. (Es geht also nicht um das Berzeviczy-Album, das im Göttinger Stadtarchiv verwahrt wird und auch im IAA publiziert wurde.) Klára Berzeviczy mag deswegen mit dem (übrigens ziemlich informativen) Auktionskatalog gearbeitet haben, sie konnte aber dadurch kein Haller-Zitat finden. Im Cornides-Album hat sie aber deren zwei gefunden. Eines auf Seite 82v, dieses konnte sie aber nicht identifizieren auch ich habe dies vergebens versucht -, was vermutlich bedeutet, dass entweder die Quellenangabe des Einträgers fehlerhaft ist (,Haller!“), oder das tatsächlich von Haller stammende (einige Wörter lange) Zitat aus einem prosaischen Werk des schweizerischen Autors stammt. Das andere Zitat ist auf Seite 143r zu lesen und stammt aus dem Gedicht An Se. Excellenz Herrn Gerlach Adolf v. Münchhausen. . . Es geht um ein Autograph von 1755, das in Bezug auf Haller also relativ früh entstanden ist. Der Einträger, Christoph Gottlieb Hofmann, stammt sicherlich nicht aus Ungarn. Berzeviczy hat in den verbliebenen fünf Stammbüchern insgesamt vier Haller-Zitate nachgewiesen. Diese Zahl ist um sechs weniger als die Zahl der Zitate, die wir bezüglich derselben Alben im IAA zugänglich gemacht haben. Ein (allerdings kein alleiniger) Grund dafür ist, dass Berzeviczy das Album von Sándor Podmaniczky aus der Textausgabe von Wilhelm Ebel untersucht hat, die nicht vollständig ist. Göttinger Studenten-Stammbuch aus dem Jahre 1786: In Auswahl herausgegeben und mit einem Vorwort versehen von Wilhelm EBEL (Göttingen: Vandenhoeck \& Ruprecht, 1966). Vgl. Klára BERZEVICZY, „Ungarische Peregrinus-Stammbücher als Vermittler der deutschen Literatur in Ungarn. Ein Beitrag zu den deutsch-ungarischen kulturellen Beziehungen“, in Mittlerin aus Europas Mitte: Fundamente und Perspektiven der deutschen Sprache und ihrer Literatur im ostmittel-und südosteuropäischen Raum: Beiträge des III. Kongresses des Mitteleuropäischen Germanistenverbandes (MGV) in Wien, 8.-10. April 2010, Carolin sollfranK, Johann WELLNER (Hg.), Veröffentlichungen des Mitteleuropäischen Germanistenverbandes 3, 27-41 (Dresden: Thelem, 2017). Ich hatte die Möglichkeit, diesen Aufsatz von Klára Berzeviczy noch vor dessen Erscheinung zu lesen, wofür ihr und Péter Lökös mein Dank gebührt.
}

${ }^{15}$ OSZK, Oct. Lat. 117., p. 31r., 44v., 45v. Vgl. BERZEviCZY-LÖKÖs, „Zitate deutscher. . “, 148-150; und IAA, 7,770., 7,778., 7,780. Ich würde bezüglich der Personen der Einträger bemerken, dass der vollständige Name von „H. W. Maurer“ Heinrich Wilhelm Maurer und der von „F. W. Volkmar“ richtig Friedrich Wilhelm Vollmar wäre. Vgl. Die Matrikel der Universität Tübingen. Band III. 1710-1817, bearbeitet von Albert BÜRK und Wilhelm wILLE (Tübingen: Universitätsbibliothek Tübingen, 1953), 211., 36,351 und 202., 36,127.

${ }^{16}$ IAA, 7869., 7797. Im Fall des Vorigen ist der Einträger, Christian Karl Philipp Vollmar, offenbar mit Friedrich Wilhelm Vollmar verwandt, der ebenfalls Haller zitiert. BÜRK-WILle, Die Matrikel. ., 202., 36,128. Vgl. Fußnote 15.

${ }^{17}$ IAA, 7,739., 7,828.

${ }^{18}$ IAA, 7,731., 7,800., 7,744., 7,783., 7,784., 7,865.

${ }^{19}$ Register zur Matrikel der Universität Erlangen 1743-1843, bearbeitet von Kurt WAGNER. Mit einem Anhang: Weitere Nachträge zum Altdorfer Personenregister von Elias von steInMEYeR (München-Leipzig: Duncker \& Humblot, 1918), 42. 1766.09.18. Vgl. IAA, 7,783. Die zitierte Textstelle: Ode an einen entfernten Bruder, 123-124. 
(„Hubers-Gedichte“). ${ }^{20}$ Die insgesamt 13 Zitate im Album von Coellnberger stammen ausnahmslos von Studenten: Sie sind in den meisten Fällen in Tübingen und in Erlangen entstanden. Lediglich zwei von ihnen waren Landsleute des Besitzers, wie Johann Gottlieb Klein, der in Siebenbürgen geboren wurde (auf seinen Eintrag haben auch die beiden Autoren Bezug genommen), ${ }^{21}$ sowie der Medizinstudent Sámuel Lischoviny ${ }^{22}$ aus Neusohl.

Die Autoren machen natürlich keinen definitiven Unterschied zwischen ungarischen und nicht ungarischen Einträgern in ihrem Aufsatz. ${ }^{23}$ Wenn wir bedenken, dass das Ziel ihrer Untersuchung war, die ungarische Rezeption der deutschen Dichtung des 18. Jahrhunderts anhand von Stammbüchern zu beschreiben, muss ihr Vorgehen teils für angebracht gehalten werden. Es ist in der Tat wissenswert, dass zum Beispiel die Umgebung der ungarischen Studenten, die Mitte der 1760er Jahre in Tübingen studiert haben, die Dichtung von Haller hoch geschätzt hat. ${ }^{24}$ Es ist auch tatsächlich so, dass in den von uns untersuchten Alben die Mehrheit der ausländischen Einträger, die Haller zitieren, in einer der Universitätsstädte, die auch von ungarischen Studenten frequentiert wurden, den schweizerischen Dichter angeführt haben. Bei der derzeitigen Aufarbeitung geschah dies in den meisten Fällen, jeweils fünfmal gerade in Tübingen, weiters noch in Göttingen (wo Haller zwischen 1736 und 1753 als Professor der Universität tätig war). Bei der Auswertung dieser Daten muss auch in Betracht gezogen werden, dass die meisten ausländischen Einträger immerhin im Ausland in die Stammbücher der Ungarn geschrieben haben, vor allem in die Stammbücher der Peregrini, die sie an der Universität getroffen haben. So oder so, aus der Sicht der ungarischen Rezeption ist es dennoch nicht völlig uninteressant, ob ein Auszug aus dem Gedicht Die Tugend von Haller im Eintrag von Johann Reinhold Forster (dem gelehrten Reisebegleiter des berühmten Entdeckers James Cook) oder von Susanna Catharina Binder ins Stammbuch eines jungen ungarischen Mannes geraten ist. Der Erstere im Mai 1786 in Halle, ${ }^{25}$ der Letztere im Juli 1784 in Bistritz. ${ }^{26}$ Es ist also einerseits tatsächlich wichtig, die Daten zu sammeln, die aus den Einträgen von Ausländern in

\footnotetext{
${ }^{20}$ BÜRK-WILLE, Die Matrikel. ., 201., 36,088. Vgl. IAA, 7,828. Die zitierte Textstelle: Die Vorsicht, 1-12.

${ }^{21}$ SZABÓ Miklós - SZÖGI László, Erdélyi peregrinusok: erdélyi diákok európai egyetemeken 1701-1849 [Siebenbürger Peregrinanten: Siebenbürgische Studenten an europäischen Universitäten 1701-1849] (Marosvásárhely: Mentor Kiadó, 1998), $2,117$.

${ }^{22}$ Bei seinem Eintrag fehlt der Ort. Er hat übrigens ab 1765 an der Wiener Universität studiert. Vgl. KISSNÉ BOGNÁR Krisztina, Magyarországi diákok a bécsi tanintézetekben 1526-1789 [Ungarische Studenten an Wiener Lehranstalten]. Magyarországi diákok egyetemjárása az újkorban 13. [Auslandsstudien ungarischer Studenten in der frühen Neuzeit 13] (Budapest: Eötvös Loránd Tudományegyetem Levéltára, 2004), 4,619. Im Lexikon von Szinnyei taucht er unter dem Namen Sámuel Dávid Lischoviny auf. Vgl. SzINNYei József, Magyar írók élete és munkái [Leben und Werk ungarischer Schriftsteller], 14 Bde. (Budapest: Hornyánszky Viktor, 1891-1914), 7:1,287. Lischoviny zitiert übrigens von Uz: Ode an die Weisheit, 37-42.

${ }^{23}$ Aus den zur Ausgabe beigelegten biographischen Informationen, die im Fall der Einträger aus Ungarn meist die Daten aus dem Lexikon von Szinnyei wiedergeben, stellt sich natürlich die Herkunft der Einträger heraus, dies hat aber, die Ganzheit der Arbeit betrachtet, überhaupt keine Konsequenz. In den zusammenfassenden Tabellen sind derartige Daten nicht zu finden.

${ }^{24}$ Aus der Periode zwischen 1760 und 1770 sind die Namen von 36 ungarischen Studenten bekannt, die in Tübingen immatrikuliert wurden. TAR, Magyarországi diákok. . . [Ungarische Studenten. . .], 3,018-3,053.

${ }^{25}$ IAA, 8,687.

${ }^{26}$ IAA, 12,094 .
} 
Alben der Hungarica-Art herausgelesen werden können, andererseits aber lohnt es sich, sie getrennt zu behandeln.

Wenn wir die auf diese Weise sortierten Einträge betrachten, bekommen wir folgendes Ergebnis: Im analysierten Stoff erscheinen etwa ab Mitte der 1750er Jahre Zitate des Dichters, der 1732 debütierte und auf einen Schlag bekannt wurde. Die Zahl der Zitate wächst ständig bis 1790, im letzten Jahrzehnt des Jahrhunderts verschwindet aber der schweizerische Dichter völlig aus den Einträgen von Ausländern, während er von den Ungarn - obwohl einigermaßen seltener - auch damals noch mit Vorliebe zitiert wurde. Ich würde auch noch hinzufügen, dass die analysierte Stichprobe nicht unbedingt klein ist: In den Einträgen von Ausländern wurden insgesamt in 29 Fällen, ${ }^{27}$ in Autographen von ungarischen Einträgern in 24 Fällen Haller-Zitate gefunden. $^{28}$

Es lohnt sich, all dies mit den Daten der Datenbank Repertorium Alborum Amicorum (RAA) von Werner Wilhelm Schnabel zu vergleichen. ${ }^{29}$ In der Datenbank von Schnabel kann derzeit auf die Grunddaten von mehr als 227,000 Stammbucheinträgen zugegriffen werden. Schnabel hat diese in den meisten Fällen aus der Fachliteratur übernommen. Wenn zum Beispiel einer Textausgabe, einer Datenbank, eines Katalogs oder sogar eines Fachaufsatzes entnommen werden kann, dass in einem der Einträge ein Haller-Zitat zu lesen ist, erscheint diese Information theoretisch auch im RAA, das derzeit 274 Einträge verzeichnet, die Haller-Zitate enthalten. ${ }^{30}$ Ihre zeitliche Verteilung sieht folgendermaßen aus:

\begin{tabular}{|l|c|}
\hline Periode & Albrecht von Haller-Zitate in der RAA \\
\hline $1740-1749$ & 21 \\
\hline $1750-1759$ & 39 \\
\hline $1760-1769$ & 84 \\
\hline $1770-1779$ & 82 \\
\hline $1780-1789$ & 42 \\
\hline $1790-1800$ & 6 \\
\hline
\end{tabular}

${ }^{27}$ Chronologisch: IAA, 9,631.*, 10,964.*, 7,778., 7,780., 7,869.*, 7,797.*, 6,171., 6,166.*, 11,704.*, 11,693.*, 8,308., 11,658., 11,692., 6,150.*, 4,792.*, 7,646.*, 9,465., 9,227. , 4,233.*, 10,453.*, 8,687.*, 8,930.*, 8,905., 8,965., 8,879. $, 9,161 .^{*}, 8,933$., 9,543., 3,073. Der * nach den Rekordzahlen verweist darauf, dass der Autor selbst auf keine Weise die Quellen des zitierten Textes angegeben hat.

${ }^{28}$ Chronologisch: IAA, 2,454., 7,770., 11,698., 2,862., 8,125., 11,218.* 9,373.*, 6,045.*, 8,285.* , 9,554. , 12,094.*, 12,120.*, 12,219.*, 7,341., 9,108., 3,103.*, 8,065., 11,530.*, 11,379., 10,000.*, 3,368.*, 5,228.*, 8,069., 5,266.* Zur Wahrheit gehört natürlich, dass einer der erwähnten Einträge (IAA, 8,285.) im Album des Mecklenburger Georg Ludolph von Behr, also im Stammbuch eines deutschen Besitzers, erhalten geblieben ist, er stammt aber von einem jungen Käsmarker, Christian Genersich. Über Behr siehe: Die Matrikel der Georg-August-Universität zu Göttingen 1734-1837, herausgegeben von Götz von SELLE (Leipzig: A. Lax, 1937), 10,559. Ein anderes Autograph (IAA, 8,125.) ist aus dem Album des Münzenberger Johann Philipp Wiesener bekannt: Es geht auch diesmal um den Eintrag eines Studenten aus Ungarn, József Podmaniczky. Über Wiesener siehe: SELle, Die Matrikel. ., 9,913.

${ }^{29}$ Werner Wilhelm SCHNABEL, Repertorium Alborum Amicorum, 1998-2017; http://www.raa.phil.uni-erlangen.de.

${ }^{30}$ Zuletzt gesehen am 10. Juli 2017. 
Anhand dieser Daten lässt sich zeigen, dass Haller noch früher, nämlich in den $1740 \mathrm{er}$ Jahren, in die Alben geraten ist. Im darauffolgenden Jahrzehnt wächst die Zahl der ihn zitierenden Autographe beträchtlich, sie verdoppelt sich praktisch. Der Prozess stagniert auch in den 1760er Jahren noch nicht, die Zahl der Zitate nimmt sogar radikal zu: Sie verdoppelt sich wieder und bleibt auch im nächsten Jahrzehnt auf diesem Wert. Der Rückfall beginnt erst später, in den 1780er Jahren, und der Dichter verschwindet zum Ende des Jahrhunderts aus den Alben. Es ist offensichtlich, dass die Zahlen aus dem RAA nicht exakt sein können, ausgehend von der Größe des aufgearbeiteten Stoffes könnten sie aber die Grundtendenzen theoretisch schon anzeigen.

In der Fachliteratur über Stammbücher kann heute aber schon als Gemeinplatz betrachtet werden, dass solche Zahlen an sich uninterpretierbar sind, da es überhaupt nicht egal ist, wie groß der Stoff war, dem sie entnommen wurden. Es ist aus dem RAA jedoch nicht ersichtlich, wie viele Einträge Schnabel genau aufarbeiten musste, um die (sagen wir mal) aus den 1740er Jahren registrierten 21 Haller-Zitate zu detektieren, ob mehr oder aber viel weniger als zur Angabe der 39 Zitate aus den 1750er Jahren. ${ }^{31}$ Die deutschen Forscher rechnen dementsprechend anders und so entsteht in der Tat ein anderes Ergebnis. Zum Beispiel bei Horst Steinhilber, der sein Buch über die Mentalität der Studenten der 1740-1800er Jahre gänzlich auf der Grundlage von Informationen geschrieben hat, die den Stammbüchern zu entnehmen sind. ${ }^{32}$ Während seiner Arbeit hat er beinahe 300 Alben untersucht und von den 14 meistzitierten Autoren hat er 1598 Zitate registriert; ${ }^{33}$ von Haller insgesamt 85. Die frühesten stammen aus den 1750er Jahren und bezüglich der erwähnten 14 Autoren und des gegebenen Jahrzehntes sind diese beinahe $8 \%$ des Materials, das heißt - gemäß der Feststellung Steinhilbers -, dass diese zu dieser Zeit den höchsten Anteil haben. Die Popularität Hallers, die in Alben zu messen ist, hat danach kontinuierlich abgenommen. In den 1760er Jahren nur ein bisschen, aber in den zwanzig Jahren zwischen 1770 und 1790 schon bedeutend: Ihr Anteil ist auf etwa die Hälfte der früheren gefallen. Um die 1790er Jahre ist der schweizerische Dichter fast gänzlich aus den Alben verschwunden $\left(,, 1.43 \%\right.$ “). ${ }^{34}$ Katrin Henzel ist auch zu einem ähnlichen Ergebnis gekommen, während sie die Leipziger Einträge aus der Zeit 1760-1804 der Albumsammlung der Leipziger Universitätsbibliothek untersucht hat. Für ihre Monographie hat sie insgesamt 1,012 Einträge verwendet und in den untersuchten Autographen konnte sie den Autor der zitierten Texte in 569 Fällen identifizieren. Haller-Zitate hat sie in 19 Einträgen gefunden. Aufgrund ihrer Berechnungen und bezüglich der identifizierten Zitate lag die Popularität Hallers in den 1760er Jahren bei 5.9\%, im nächsten Jahrzehnt bei 3.9\%, in den zehn Jahren zwischen 1780 und 1789 fiel diese auf $2.3 \%$ und ab diesem Zeitpunkt konnte sie die Gedichte des schweizerischen Dichters in den untersuchten Alben gar nicht mehr nachweisen. ${ }^{35}$

Es ist ersichtlich, dass diese Untersuchungen tatsächlich zu einem anderen Ergebnis geführt haben, als es die zeitliche Verteilung der Anzahl der besprochenen Zitate an sich ahnen ließ.

\footnotetext{
${ }^{31}$ Vgl. Katrin HENZEL, Mehr als ein Denkmal der Freundschaft: Stammbucheinträge in Leipzig 1760-1804. Literatur und Kultur. Leipziger Texte - Reihe B: Studien 4 (Leipzig: Leipziger Universitätsverlag, 2014), 353-354.

${ }^{32}$ Horst steinhilber, Von der Tugend zur Freiheit: Studentische Mentalitäten an deutschen Universitäten 1740-1800. Historische Texte und Studien 14 (Hildesheim: Georg Olms Verlag, 1995).

${ }^{33}$ Ebd., 335. (Es geht eigentlich um 13 Autoren und die Bibel.)

${ }^{34}$ Ebd., 347.

${ }^{35}$ HENZEL, Mehr als. .., 360.
} 
Daher tauchte Haller also am häufigsten in den 1750er und 1760er Jahren in den Alben auf, darauffolgend hat seine Popularität allmählich nachgelassen und im letzten Jahrzehnt des Jahrhunderts ist sie praktisch gleich Null. Wenn wir mit den Daten, die wir gefunden haben, ähnliche Berechnungen anstellen, bekommen wir folgendes Ergebnis:

\begin{tabular}{|l|c|c|}
\hline \multicolumn{3}{|l|}{ Albrecht von Haller-Zitate in der IAA } \\
\hline Periode & von Einträgern aus Ungarn & von ausländischen Einträgern \\
\hline $1750-1759$ & $0.20 \%$ & $0.22 \%$ \\
\hline $1760-1769$ & $0.12 \%$ & $1.80 \%$ \\
\hline $1770-1779$ & $0.46 \%$ & $1.77 \%$ \\
\hline $1780-1789$ & $1.12 \%$ & $1.45 \%$ \\
\hline $1790-1800$ & $0.48 \%$ & $0 \%$ \\
\hline
\end{tabular}

*Die Prozentzahlen habe ich anhand der Zahl der Inskriptionen festgestellt, die im gegebenen Jahrzehnt von ungarischen bzw. ausländischen Einträgern stammten. Diese Werte entsprechen also auf direkte Weise weder den Daten von Steinhilber noch denen von Henzel, die Grundtendenzen zeigen sie aber genauso. (Die Zahl der untersuchten Einträge in den Jahrzehnten der Tabelle schwankte übrigens zwischen 1,436 und 273. Der Durchschnitt pro Jahrzehnt lag bei 711 Einträgen.)

Unsere Berechnungen decken sich im Fall der ausländischen Einträger auch nicht ganz mit den Ergebnissen der deutschen Forscher, da wir für die 1750er Jahre einen ausgesprochen niedrigen Wert erhalten haben (was sogar die Konsequenz irgendeiner Messanomalie sein kann), obendrein ist der immerhin kontinuierliche und feststellbare Rückfall, der im Zeitraum 1760-1790 nachgewiesen wurde, auch nicht so auffällig. Angesichts des letzten Jahrzehntes des Jahrhunderts stimmen die Ergebnisse natürlich mit den Feststellungen Steinhilbers und Henzels vollkommen überein. Bezüglich der Haller-Zitate der Einträger aus Ungarn können wir aber in der Tat spektakuläre Unterschiede beobachten. Hallers Zitiertheit nimmt in ihren Einträgen nach 1760 nicht ab, sie wächst sogar radikal und es ist sehr auffallend, dass sie auch im letzten Jahrzehnt des Jahrhunderts einen ganz hohen Wert hat.

Wenn diese Daten wirklich ein relevantes Bild zeigen, können wir etwa folgende Feststellungen machen: Die Prozentwerte bezüglich der ungarischen Einträger der Haller-Zitate sind immer niedriger als jene bezüglich der ausländischen, und auch deswegen ist es auffällig, dass die zwei Werte in den 1780er Jahren beinahe gleich sind (1.12\% und 1.45\%). Wenn wir den (teils auch von uns bewiesenen) Berechnungen Steinhilbers und Henzels glauben dürfen, war der Kult Hallers in Alben auf deutschen Gebieten intensiver und kurzlebiger. Im Fall der ungarischen Einträger können wir ein anderes Muster beobachten. Das Ganze hat sich später entfaltet, wurde in der fraglichen Periode immer stärker und hat auch länger gedauert. Es gibt aber noch einen Unterschied, der wichtig zu sein scheint und der auch unseren Daten zu entnehmen ist, und zwar: Gut ein Drittel der Haller-Zitate in Ungarn stammt von Frauen, ${ }^{36}$ während die ausländischen Einträger ausnahmslos Männer sind.

\footnotetext{
${ }^{36}$ Es geht um insgesamt 9 Einträge. Chronologisch: IAA, 11,218.*, 9,373.*, 6,045.*, 12,094.*, 12,120.*, 11,530.*, 5,228.*, 8, 069., 5,266.* Der * nach den Rekordzahlen verweist auch hier darauf, dass der Einträger die Quellen des zitierten Textes nicht angegeben hat.
} 


\section{FRAUEN}

Letzteres ist auf den ersten Blick eigentlich gar nicht überraschend. Es galt als eine intime Sache, eine Frau darum zu bitten, einen Stammbucheintrag zu schreiben und umgekehrt genauso. Der Teil aus dem Tagebuch von Antónia Kölcsey ist bekannt, in dem eine Familienstreiterei von 1843 erzählt wird, deren Grund war, dass sie ihr Album einem „Fremden“ „,ausgeliefert“ hat. $^{37}$ Die ungarischen Peregrini haben während ihrer Studienreisen relativ selten eine dermaßen vertraute Beziehung mit Frauen gepflegt. Auch ein anderes Stammbuch von Samuel Coellnberger ist hier als Beispiel erhalten geblieben. Dieses hat er direkt vor seiner Peregrination, 17611762, vermutlich während seines Albisierens geführt, und in diesem Buch sind 8 Autographe von Frauen zu finden, ${ }^{38}$ in jenem Album hingegen, das er während seiner Peregrination benutzt hat, keine. Anscheinend war die Sache jedoch nicht ausschließlich davon abhängig. Unter den 274 Haller-Zitaten im RAA stammen nämlich insgesamt 3 von Frauen, ${ }^{39}$ während Katrin Henzel im von ihr untersuchten Material keine gefunden hat. Ihr zufolge kann dies mit dem eigenartigen Inhalt der philosophischen Lehrgedichte Hallers erklärt werden, ${ }^{40}$ aber angesichts der Haller-Zitate der ungarischen Frauen scheint ihre Argumentation nicht richtig zu sein. Dies könnte (theoretisch) sogar bedeuten, dass wir uns nicht nur den zeitlichen Verlauf, sondern auch die Szene des ungarischen Kults Hallers in Alben anders vorstellen müssen als dies die deutschen Experten während der Erschließung der von ihnen untersuchten Einträge skizziert haben.

Auf dieser Grundlage könnten wir auch meinen, dass in der ungarischen Haller-Rezeption auch die Frauen eine wichtige Rolle gehabt haben. Ihre Einträge sind in Ungarn fast ausnahmslos in deutschen Städten entstanden. ${ }^{41}$ Es gibt nur einen Eintrag aus Siebenbürgen (aus Bistritz), die anderen wurden in Ödenburg bzw. in den deutschen Städten der Zips, in Leutschau, in Zipser Neudorf, in Käsmark geschrieben, es gibt aber auch ein Haller-Zitat aus Osgyán. Weitgehende Folgerungen können daraus natürlich nicht gezogen werden, zum Beispiel, weil aus der Periode zwischen 1750 und 1800 bisher kaum einige Einträge von siebenbürgischen Frauen aufgearbeitet worden sind. Es kann eher auffallen, wie groß das Gebiet ist, über das die Siedlungen zerstreut sind, aus denen die untersuchten Autographe stammen. Aus derselben Hinsicht mag interessant sein, dass jeder Eintrag von jemand anderem stammt und dass auch die zeitliche Verteilung der Einträge relativ gleichmäßig ist $-\mathrm{d}$. h. hinsichtlich der zwei Jahrzehnte, in denen sie überhaupt in die Alben geschrieben wurden. Sie wurden nämlich alle relativ

\footnotetext{
${ }^{37}$ Nämlich Gábor Horváth, der „,beinahe eine Woche hier verbracht hat“. Vgl. köLCSEY Antónia naplója [Das Tagebuch der Antónia Kölcsey], mit Einführung herausgegeben von Kozocsa Sándor (Budapest: Rózsavölgyi és Társa, 1938 ), 131. Zitiert durch HÁSz-FEHÉR Katalin, Elkülönülő és közösségi irodalmi programok a 19. század első felében (Fáy András irodalomtörténeti helye) [Individuelle und gesellschaftliche literarische Programme in der ersten Hälfte des 19. Jahrhunderts (András Fáy in der Literaturgeschichte)], Csokonai Könyvtár (Bibliotheca Studiorum Litterarium) 21. (Debrecen: Debreceni Egyetem Kossuth Egyetemi Kiadója, 2000), 201-202.

${ }^{38}$ OSZK, Oct. Lat. 111. IAA, 6,349., 6,337., 6,341., 6,370., 6,207., 4,938., 4,955., 4,972. Unter den Einträgen enthalten drei weder Zeit noch Ort. Die 8 Einträge sind auf Deutsch bzw. auf Ungarisch geschrieben worden.

${ }^{39}$ Es geht um folgende Einträge: M. C. Bauderin, Altdorf, 1758 (1755_weber, 12); Johanna Maria Keidel, Göttingen, 1774 (1770_wehrs1, 43); Augusta Amalia Sammet, Lipcse, 1780 (1779_sammet, 2).

${ }^{40}$ HENZEL, Mehr als. . ., 361-363.

${ }^{41}$ Obwohl zwei Frauen den Ort des Eintrags nicht angegeben haben: IAA, 11,530., 5,228.
} 
spät, in der Zeit zwischen 1779 und 1799, geschrieben, was den Eindruck wecken kann, dass gerade die Frauen im erwähnten späten Weiterleben des Haller-Kults die Schlüsselrolle gespielt haben mögen. Übrigens handelt es sich um typische Fraueneinträge auch in dem Sinn, dass wir in acht von den neun Autographen gar keinen Hinweis auf den Autor des zitierten Textes finden, das heißt, beinahe alle Texte sind ungekennzeichnet.

Die einzige Ausnahme ist der Eintrag von Amalia Trangus (Trangous), in dem ein eindeutiger Quellenhinweis zu finden ist (,Haller“). ${ }^{42}$ Das Autograph von Fräulein Trangus ist im Stammbuch von Mózes Schmidt erhalten geblieben, das aus der Periode 1787-1797 insgesamt 67 Einträge bewahrt hat. ${ }^{43}$ Es handelt sich auch diesmal um ein Peregrinationsalbum. Sein Besitzer hat das leere Büchlein wohl nicht lange vor seinem Aufbruch gekauft und gleich begonnen, Autographe zu sammeln - wie auch andere dies oft taten. So konnten 22 Inskriptionen noch in Ungarn in das Album gekommen sein, teils aus Ödenburg und Pressburg, größtenteils aus Rosenau datiert. Schmidt hat während seiner Peregrination 1787-1789 sogar von zwei Frauen Einträge erhalten (in Dresden und innerhalb von fünf Tagen), ${ }^{44}$ aber es ist bezeichnend, dass die Zahl der Frauen, die in Ungarn ins Album geschrieben haben, bei neun liegt. Nach seiner Heimkehr hat er sein Album eigentlich nur noch einmal hervorgeholt, acht Jahre später, um vierer Personen willen. Eine von diesen war Amalia Trangus. Ihr Autograph ist am 5. Dezember 1797 in Zipser Neudorf entstanden, genauso wie das von ihrer Mutter, Barbara Reinländer (,von [...] Barbara Trangouss gebornen Reinländerin“) ${ }^{45}$ und ihrer Schwester, Juliana Trangus. ${ }^{46}$ Am Tag davor hatte auch ihr Bruder, Joseph Trangus, das Stammbuch bekommen. ${ }^{47}$ Er war damals erst 15 Jahre alt, er wurde nämlich am 14. März 1783 geboren. Károly Pákh erwähnt über ihn, dass er sein ganzes Leben lang ,der lateinischen Sprache und den Geisteswissenschaften sehr zugetan war und er hat in seinen letzten Jahren leidenschaftlich die Werke von Schopenhauer gelesen“, sowie dass er sich „oft mit den Fragen der Frauenerziehung beschäftigt hat, und weil er überzeugt von deren großen Wichtigkeit war, hat er sein Haus einer zu gründenden evangelischen Mädchenschule hinterlassen“. ${ }^{8}$ Amalias Vater, Michael Johann Trangus, der um 1767 nach Zipser Neudorf gezogen ist, hat zunächst als Schriftführer und Notar, später, 1799-1804, als Oberrichter eine wichtige Rolle im Leben der Stadt gespielt. ${ }^{49}$ Nachdem 1772 die an Polen verpfändeten Städte - unter anderem Zipser Neudorf - wieder eingegliedert worden waren, wollte die Landvogtei diese Siedlungen als Schatzkammergut einstufen. Diese Bemühungen scheiterten am energischen Widerstand von Zipser Neudorf.

\footnotetext{
${ }^{42}$ IAA, 8,069.

${ }^{43}$ OSZK, Oct. Lat. 1,247. Über den Besitzer siehe: TAR, Magyarországi diákok. . . [Ungarische Studenten. . .], 1,360.

${ }^{44}$ IAA, 8,029., 8,074.

${ }^{45}$ IAA, 8,067. Vgl. PÁKH Károly, Az iglói Ág. H. Ev. Fógymnasium története [Geschichte des evangelischen Obergymnasiums A. B. in Iglau] (Igló: Schmidt József, 1896), 48; und HADOBÁs Sándor, „Trangous Lajos, a reformkor sikeres bánya- és kohóvállalkozója“ [Lajos Trangous, der erfolgreiche Unternehmer im Bergbau- und Hüttenwesen] Bányászattörténeti Közlemények 5 (2010): 76-85, 77.

${ }^{46}$ IAA, 8,068. Vgl. NAGY Iván, Magyarország családai czímerekkel és nemzékrendi táblákkal [Ungarns Familien mit Wappen und genealogischen Tabellen], 12 Bde. (Pest: Ráth Mór, 1857-1868), 11:326.

${ }^{47}$ IAA, 7,499.

${ }^{48}$ РÁKH, Az iglói... [Geschichte des evangelischen Obergymnasiums. . .], 48-49.

${ }^{49}$ Ebd., 48.; HADOBÁs, „Trangous Lajos. ..“ [Lajos Trangous...], 76-77.
} 
Die Stadt hatte nämlich gerade in einer Schrift von Michael Johann Trangus - zuletzt erfolgreich - gegen das Verfahren protestiert. ${ }^{50}$ Es handelt sich hier um eine vermögende Familie und es ist kennzeichnend, dass nach dem Tod der Brüder Trangus (Ludwig im Jahre 1855, Joseph im Jahre 1861) deren Stiftungen die evangelische Kirche in Zipser Neudorf in eine radikal neue Situation gebracht haben. ${ }^{51}$

Michael Johann Trangus und Barbara Reinländer haben 1773 geheiratet. Wir haben keine Informationen über das Geburtsdatum ihrer Töchter, Juliana und Amalia. Sie benutzen aber in ihren Unterschriften ihre Geburtsnamen und keine von ihnen tituliert sich als verheiratet, das heißt, sie mögen nicht die ältesten der insgesamt sechs Kinder des Ehepaares gewesen sein. ${ }^{52}$ Es geht also um junge Fräulein, die Töchter eines reichen und einflussreichen Bürgers von Zipser Neudorf, eines vermögenden Unternehmers, die offensichtlich in einer Familie erzogen wurden, die Literatur geschätzt hat und die vermutlich auch in den Fragen der „Frauenerziehung“ aufgeklärt war. Ohne Zweifel haben sie aus jeder Hinsicht zur Elite der Bürgerschaft in Zipser Neudorf gehört. Im Album von Schmidt zitieren beide Schwestern von deutschen Dichtern. Amalia, wie wir gesehen haben, von Haller, Juliana ein Gedicht von Gellert. ${ }^{53}$

Es lohnt sich also zu untersuchen, ob jede Frau, die in Ungarn Haller zitiert, in so einer ausgezeichneten Situation war, und noch wichtiger, von welcher Kenntnis des schweizerischen Dichters ihre Zitate tatsächlich zeugen.

\section{ELEONORA DONNER, SOPHIA ENGELMAYER UND DAS „SECHSZEILIGE“ AUS ÜBER DIE EHRE}

Das früheste von einer Frau eingetragene Haller-Zitat stammt von der Ödenburgerin Eleonora Donner (,Eleonora Donnerin“). ${ }^{54}$ Ihre Person betreffend können wir uns lediglich auf das Stammbuch mit ihrem Eintrag (im Album von István Márton) verlassen. ${ }^{55}$ In diesem Buch ist nämlich eine Inscriptio eines Johann Sigismund Donner zu lesen, vom 4. Mai 1779, aus Ödenburg. ${ }^{56}$ Eleonora hat nur wenige Tage später, am 10. des Monats, das Stammbuch in die Hände genommen, ebenfalls in Ödenburg, es ist also wahrscheinlich, dass sie verwandt, sogar nahe Verwandte waren. Verlässliche Informationen haben wir nur über die Person von Johann Sigismund, der sich am 15. Oktober 1779 zusammen mit Márton an der theologischen Fakultät der Universität in Göttingen immatrikuliert hat. ${ }^{57}$ Sigismund Donner hat aber

${ }^{50}$ BRUCKNER Győző, „Igló kir. korona- és bányaváros története“ [Geschichte der Königlichen Frei-und Bergstadt Iglau], in Iglói diákalbum, hg. von MAKRA Zoltán und MOHR Győző, 42-81 (Budapest: Iglói Főgimnázium, 1929 ), 67.

${ }^{51}$ PÁKH, Az iglói... [Geschichte des evangelischen Obergymnasiums. . .], 47-48.

${ }^{52}$ Ebd., 48.

${ }^{53}$ Die zitierten Gedichte: Albrecht von Haller, Gedanken bei einer Begebenheit, 13-16; und Christian Fürchtegott Gellert, Zufriedenheit mit seinem Zustande, 9-12.

${ }^{54}$ IAA, 11,218. Die zitierte Textstelle: Über die Ehre, 217-222.

${ }^{55}$ OSZK, Oct. Lat. 1222. Berzeviczy und Lőkös haben das Album untersucht, sie haben aber das Zitat von Eleonora Donner, das keine Quellenangabe enthält, nicht registriert.

${ }^{56}$ IAA, 8,173.

${ }^{57}$ TAR, Magyarországi diákok... [Ungarische Studenten...], 678, 680. 
eigentlich Medizin studiert. Im zweiten Jahrgang der Zeitschrift Tudományos Gyüjtemény kommt sein Name vor: György Hrabowszky listet ihn unter den „Gelehrte[n] geboren im Komitat Ödenburg“ auf („Donner János Zsigmond Soproni fi 1784“). ${ }^{58}$ Die Jahreszahl 1784 verweist offensichtlich auf die in Göttingen erschienene Arbeit von Donner. ${ }^{59}$ Jenő Házi informiert uns auch über die Eltern des ,gelehrten“ Arztes. ${ }^{60}$ Laut seiner Daten war sein Vater, Johann Donner (,Donner János“) Eisenhändler, der im Juli 1743 die Tochter von „Leitner Ferenc“, externem Ratsmitglied und Eisenhändler, geheiratet hatte, namentlich „Eleonóra“, die zu der Zeit des gegenständlichen Eintrags, 1779, schon seit 16 Jahren Witwe war. Theoretisch kann auch sie selbst jene „Eleonora Donnerin“ sein, die das Haller-Gedicht zitiert, es ist aber einigermaßen seltsam, dass sie weder auf ihren Geburtsnamen noch auf ihren Familienstand verweist.

Von nun an können wir wirklich nur raten: Eleonora Leitner könnte sogar eine Tochter mit dem Namen Eleonora gehabt haben, die auf diese Weise natürlich als die Schwester von „Donner János Zsigmond Sopron fi“ (und nicht als seine Mutter) die Zeilen aus Über die Ehre zitiert haben kann. Wenn es um so etwas geht - was am meisten die einfache Tatsache wahrscheinlich macht, dass im Stammbuch von Márton das Namenszeichen von Johann Sigismund tatsächlich $\mathrm{zu}$ finden ist, der $\mathrm{zu}$ der Zeit noch vor seinem Göttinger Studium stand -, ${ }^{61}$ dann stammte Eleonora Donner ebenfalls aus den vermögenden Schichten der Ödenburger Bürgerschaft, obwohl ihr Mann (oder Vater), der als externes Ratsmitglied 1763 gestorben ist, bei Weitem nicht so einflussreich war, wie der Vater von Amalia Trangus, der auch Oberrichter war. Es charakterisiert gut die finanzielle Situation der Familie - und vielleicht ihre Zuneigung zur Bildung -, dass Johann Sigismund ab Ende 1779 jahrelang in Göttingen studieren konnte.

Das Autograph von Eleonora Donner kann übrigens auch nicht als typischer Fraueneintrag betrachtet werden, nämlich in der Hinsicht, dass er überraschenderweise auch ein Symbol enthält, ${ }^{62}$ was in der Tat äußerst selten in Einträgen von Frauen vorkommt. Das Haller-Zitat an sich wäre keine so große Überraschung mehr, da der schweizerische Dichter in den Alben damals oft zitiert wurde (bekanntlich von Männern), und wir können auch der Tatsache höchstens einen symbolischen Wert zuschreiben, dass die Frau, die in Ungarn am frühesten einen solchen Text zitiert, gerade aus einer der (wohl eben vermögenderen) deutschen Familien von Ödenburg stammt. Drei Monate später geriet nämlich ein Haller-Zitat auch in Leutschau in das Album von János György Budaeus, und zwar von Sophia Engelmayer, die sich als Witwe bezeichnet. ${ }^{63}$ Und was wirklich beachtlich ist: Frau Sophia zitiert genau dasselbe Gedicht wie Eleonora Donner, obendrein genau dieselben Zeilen:

\footnotetext{
${ }^{58}$ Tudományos Gyüjtemény [Wissenschaftliche Sammlung], 1818. III. Band, 103.

${ }^{59}$ Commentationis medico-chirurgicae de gibbositate Pars I. Göttingae, 1784.

${ }^{60}$ HÁZI Jenő, Soproni polgárcsaládok 1535-1848 [Ödenburger Bürgerfamilien 1535-1848], 2 Bde. (Budapest: Akadémiai Kiadó, 1982), 1:350 [3,735-3,736].

${ }^{61}$ Natürlich gibt es auch andere Möglichkeiten. Házi berichtet in seinem Buch zum Beispiel von einem Schuster namens „Donner György“, der 1754 das Bürgerrecht erlangt hat. Seine Witwe, „Mária“, bezeichnet sich in ihrem Testament 1787 als die Witwe eines Spitalverwalters. Ebd., 1:350 [3,737].

${ }^{62}$ Das Symbol: „Trau, schau, wem.“

${ }^{63}$ OSZK, Oct. Lat. 605., IAA, 9,373.
} 
O selig, wem sein gut Geschicke

Bewaht vor grosen Ruhm und Glicke,

der, was die Welt erhebt, verlacht;

der frei von Joche der Geschäfte,

des Leibes und der Seele Kräfte

zum Werkzeug für die Tugend macht! $!^{64}$

Offenbar ist dies kein Zufall. Das heißt, weder Sophia Engelmayer noch Eleonora Donner haben das Gedicht von Haller (dessen letzte Version 240 Zeilen lang war) direkt zitiert, sondern aus einem Medium (oder aus mehreren Medien), in dem die betreffenden sechs Verszeilen als losgetrennte Einheit hervorgehoben zu lesen waren, und das gleichzeitig beliebt genug war, um bis 1779 auch zu zwei deutschen Frauen in Ungarn zu gelangen, die ziemlich weit entfernt voneinander gewohnt haben. Natürlich können wir auch an die anthologieartigen Ausgaben denken, die ausdrücklich für die Albumbenutzer zusammengestellt wurden, ${ }^{65}$ auch weil die sechs zitierten Verszeilen in mehreren dieser Sammlungen zu finden sind: einmal als losgetrennte Einheit, ein andermal als hervorgehobene Passage. Zum Beispiel im Buch von G. H. Meißner mit dem Titel Stammbuch oder Denkmähler der Freundschaft und Liebe, das 1809 schon zum zweiten Mal erschien, außerdem noch zum Beispiel 1811, 1814 und sogar 1823. ${ }^{66}$ Diese Ausgaben, die die Zitate von vornherein in Gruppen wie „Für Männer“, „Für Frauen“, „Für Jünglinge“, „Für Mädchen“ publizierten, zeigen jedenfalls an, dass das hier untersuchte Zitat gewöhnlich als eigenständige Einheit für die Albumbenutzer angeboten wurde. Es soll bemerkt werden, dass die Ausgabe den Text, den die zwei Frauen gewählt haben, Männern empfiehlt, und zwar in der Gruppe „Männer an Männer“. Dass am Anfang der 1800er Jahre die sechs Zeilen auch noch von anderen als eine für sich stehende Einheit behandelt wurde, kann auch das Opus von Friedrich Zuckschwerdt illustrieren, das ebenfalls für Albumbenutzer herausgegeben wurde. Auch in diesem Werk ist der fragliche Teil hervorgehoben zu lesen, und zwar samt Quellenangabe (,v. Haller“). ${ }^{67}$ Wir können uns aber auch auf Bücher vor 1779 beziehen, die unsere Einträger in die Hände genommen haben können. So

\footnotetext{
${ }^{64}$ In der ersten Zeile ist die Form wem offensichtlich ein Schreibfehler, es steht dort statt wen. Im Zitat von Eleonora Donner scheint das Wort wahrer aus der letzten Zeile eine noch interessantere (das Original von Haller auf sinnvolle Weise verändernde) Abweichung zu sein. (Darüber s. im Weiteren.) Im Eintrag der Ödenburger Frau sind die sechs Zeilen folgendermaßen zu lesen: „O seelig, wen sein Gut Geschicke/Bewahrt vor grossen Ruhm und Glücke,/der, was die Welt erhebt, verlacht,/der, frey vom Joche der Geschäfte,/des Leibes und der Seelen Kräfte/zum Werckzeug wahrer Tugend macht." (Hervorhebung von mir, M. L.)

${ }^{65}$ Hauptsächlich ab dem letzten Drittel des Jahrhunderts. Vgl. Werner Wilhelm SCHNABEL, Das Stammbuch: Konstitution und Geschichte einer textsortenbezogenen Sammelform bis ins erste Drittel des 18. Jahrhunderts, Frühe Neuzeit 78 (Tübingen: Max Niemeyer Verlag, 2003), 511-519.

${ }^{66}$ Stammbuch, oder Denkmähler der Freundschaft und Liebe. Zum Gebrauche für jedes Alter und alle Stände, als eine angenehme Rückerinnerung an die Jugendfreunde, hg. von G. H. MEIßNER (Leipzig-Wien: Gerold'sche Universitätsbuchhandlung, 1809), 69.

${ }^{67}$ Friedrich zUCKSCHWERDT, Sphinx und Harmonia oder Sammlung noch nirgends abgedruckter Räthsel, Charaden, Logogriphen und Anagrammen nebst einem Blumenkranze für Stammbücher (Berlin: Eigenverlag des Hg., 1813 ), 170.
} 
zum Beispiel auf das Werk von Johann Heinrich Schütte über Klever Heilquellen ${ }^{68}$ oder auf die Ausgabe eines anderen Arztes, Friedrich Boerner, die das Haller-Zitat ebenfalls getrennt behandelt, ${ }^{69}$ worauf wir natürlich auch in verschiedenen Periodika treffen können. So zum Beispiel in der dritten Nummer des zweiten Jahrganges des Dresdnischen Magazins, das von Michael Gröll ab 1760 herausgegeben wurde, ${ }^{70}$ oder in einem Aufsatz aus 1766, der ausgesprochen die Dichtkunst von Haller analysiert hat, in einem moralischen Wochenblatt mit dem Titel Der Glückselige, das ab 1763 in Halle erschien. ${ }^{71}$ Dieser anonyme Aufsatz beschäftigt sich seitenlang mit der Analyse von Über die Ehre (dieses Beispiel hinkt also ein bisschen), ${ }^{72}$ aber die sechs Zeilen, die uns hier interessieren, stechen deutlich unter den zu analysierenden (und natürlich prosaischen) Textteilen hervor. Im Lesebuch von Johann Georg Sulzer von 1768 tauchen die Verszeilen ohne jegliches Hinken auf, wieder gut von den vorigen und darauffolgenden Zitaten getrennt (die ebenfalls von Haller stammen), ${ }^{73}$ und wir können sie auch in der umgearbeiteten Ausgabe von 1771 finden. ${ }^{74}$ Es geht um Textsammlungen, die speziell für Gymnasiasten gemacht wurden, und die die offensichtliche Popularität dieser sechs Zeilen bezeugen, die so ein Eigenleben führen.

Aus welcher Quelle (oder welchen Quellen) genau die beiden Frauen aus Ungarn kopiert haben, ist nicht unbedingt so einfach zu sagen, aber wir können - zumindest im Fall von Sophia Engelmayer - raten. Meiner Meinung nach könnte die Ausgabe, die Frau Sophia in die Hände genommen hat, der zweite Band des Werkes von Johann Friedrich Tiede mit dem Titel Unterhaltungen mit Gott in den Abendstunden auf jeden Tag des Jahres gewesen sein, der vor 1779 sogar mehrmals erschienen ist. ${ }^{75}$ Unser Text kann hier folgendermaßen gelesen werden:

O selig, wen sein gut Geschicke

Bewaht vor grosem Ruhm und Glücke,

Der, was die Welt erhebt, verlacht;

Der frei von Joche der Geschäfte,

${ }^{68}$ Johann Heinrich SCHÜтTE, Amusemens des eaux de Cleve, oder Vergnügungen und Ergötzlichkeiten bey denen Wassern zu Cleve: Zum Nutzen derjenigen, welche die angenehme Gegenden und Merkwürdigkeiten besehen, oder diese MineralWasser gebrauchen wollen (Lemgo: Meyer, 1748), 241.

${ }^{69}$ Friedrich BÖRNER, Nachrichten von den vornehmsten Lebensumständen und Schriften Jeztlebender berühmter Aerzte und Naturforscher in und um Deutschland. II. Bd. 1. St. (Wolfenbüttel: Meißner, 1751), 166.

${ }^{70}$ Das Zitat ist am Ende eines Aufsatzes von Gotthelf Friedrich Oesfeld zu finden (Untersuchung der Frage: Warum schämt man sich der Fehler des Willens nicht so sehr als der Fehler des Verstandes). Dresdnisches Magazin, 1761. 3. 147159, 159.

${ }^{71}$ Die anonyme Arbeit ist im Wochenblatt in zwei Teilen, mit dem Titel Gebrauch der besten Dichter in der Philosophie erschienen. Der Glückselige, 1766. Stück 252, 177-192, Stück 253, 193-208. Das Haller-Zitat: Stück 252, 183.

${ }^{72}$ Ebd., 180-183.

${ }^{73}$ Vorübungen zur Erweckung der Aufmerksamkeit und des Nachdenkens: Zum Gebrauch einiger Classen des Königl. Joachimsthalischen Gymnasium (Berlin: Nicolai, 1768), 229.

${ }^{74}$ Vorübungen zur Erweckung der Aufmerksamkeit und des Nachdenkens. Zum Gebrauch einiger Klassen des Königl. Joachimthalischen Gymnasium (Berlin: Nicolai, 1771), 57-58.

${ }^{75}$ Zum ersten Mal 1771-1772 in Halle. Die von mir benutzte Ausgabe: Johann Friedrich TIEDE, Unterhaltungen mit Gott in den Abendstunden auf jeden Tag des Jahres. I-II. (Halle: Trampe, 1775), 2:84. 


\section{Des Leibes und der Seele Kräfte}

\section{Zum Werkzeug für die Tugend macht! ${ }^{76}$}

Dass die Quelle für die Witwe aus Leutschau in der Tat die Ausgabe von Tiede war, behaupte ich nicht etwa, weil mehrere Daten die Popularität des Werkes vom deutschen Prediger in Ungarn beweisen. József Kármán, der spätere reformierte Bischof, hat es zum Beispiel 1784 in Pressburg in ungarischer Übersetzung mit dem Titel Istennel való társalkodás az estvéli órákon. . . herausgegeben, ${ }^{77}$ und Mária Wesselényi (eine der Töchter von Polixéna Daniel), die hervorragend Deutsch sprach, reihte es auch unter ihre liebsten Lektüren ein - und aufgrund ihrer Deutschkenntnisse offenbar nicht nur in der ungarischen Version, die ein Jahr vor ihrem Tod erschien. ${ }^{78}$ Der eigentliche Grund für meine Behauptung ist die einfache Tatsache, dass im Album von János György Budaeus, das uns das Autograph von Sophia Engelmayer erhalten hat, auch ein anderer Beitrag zu lesen ist, der eindeutig aus der Ausgabe von Tiede zitiert. Dieser ist 13 Tage später, aber ebenfalls in Leutschau, entstanden; sein Einträger ist Johann Michael Schmer. ${ }^{79}$ Die von ihm zitierte Inscriptio lautet folgendermaßen:

Der Christ, der wahre Christ, der Tugend unterthan,

Sieht alles, was geschieht, durch Gott gelassen an.

Er weiss: es sey nicht er, es sey nur Gott Regente,

der das, was ihm beliebt, sehr leicht verfügen könte.

Ich konnte den Autor der zitierten Zeilen nicht identifizieren. Es kann sogar Tiede selbst sein, da das Gedicht zurzeit ausschließlich in seinem Buch zu finden ist, in auf den Buchstaben genau derselben vierzeiligen Form, die von Schmer zitiert wird, ${ }^{80}$ was überhaupt nicht überraschend ist. Schmer deutet in einer Quellenangabe nämlich eindeutig darauf hin, woher er die ominösen Verszeilen genommen hat: „Tiede“. In welcher Beziehung Sophia Engelmayer und Johann Michael Schmer zueinander standen, konnte ich nicht feststellen. Sie hatten auf jeden Fall einen gemeinsamen Bekannten (den Stammbuchbesitzer János György Budaeus), und sie beide haben in sein Album Verszeilen kopiert, die im Buch von Tiede zu lesen sind. Wir müssen aber vielleicht gar nicht an eine gemeinsame Bibliothek denken. Die Verwendung der Unterhaltungen können wir auch im Fall von anderen Stammbucheinträgen voraussetzen.

Im vorhin erwähnten Album von M. Fistrovits können nämlich zwei Einträge vom 5. Oktober 1784 aus Zipser Neudorf gefunden werden. Der eine stammt von Maria Elisabeth Liedemann (geborene Maria Elisabeth Lassgallner), der andere von einer Frau (die vermutlich

\footnotetext{
${ }^{76}$ Das Zitat von Tiede stimmt buchstäblich mit dem Original von Haller überein, das vor 1777 viele Male erschienen ist. In der Berner Ausgabe von 1777 hat Haller einen Ausdruck in der letzten Zeile schließlich geändert (darüber siehe im Weiteren).

${ }^{77}$ РЕтRIK Géza, Magyarország bibliographiája 1712-1860 [Ungarns Bibliographie 1712-1860], 5 Bde. (Budapest: Országos Széchényi Könyvtár, 1888-1892), 3:640.

${ }^{78}$ V. LÁszló Zsófia, „Daniel Polixéna, a »Magyar Minerva«: egy 18. századi nemesasszony élete és példája a halotti beszédek tükrében“ [Polixéna Daniel, die „Ungarische Minerva“. Leben und Beispiel einer Adeligen aus dem 18. Jahrhundert im Spiegel der Leichenreden], Sic Itur Ad Astra 19 (2008): 149-175, 160-161.

${ }^{79}$ IAA, 9461.

${ }^{80}$ TIEDE 1775, 2:335. Tiede behauptet übrigens selbst (im Vorwort zur ersten Ausgabe, das auch in den Editionen von 1775 erschienen ist), dass ein Teil der gereimten Einlagen von ihm stammt: „Das Motto, oder die Verse über jeder Unterhaltung, nahm ich wo ich es fand. Und fand ich kein bequemes, so machte ich es selbst.“ TIEDE 1775, 1:29.
} 
die Vornamen ihrer Mutter geerbt hat), der so ebenfalls mit der Unterschrift Maria Elisabeth Liedemann erscheint. ${ }^{81}$ Die von Frau Liedemann zitierten Zeilen sind die Folgenden:

Nur gut, wen gleich nicht gross,

Soll deine Laufban sein,

des frommen Lob ist mehr,

Als Marmor Leichen-Stein.

Die von Fräulein Liedemann zitierten Zeilen:

Was ist das Leben ohne Freunde?

Und auch, was wär es ohne Feinde?

Die Verszeilen, die von den beiden Frauen kopiert wurden, können auch in diesem Fall ausschließlich in den Unterhaltungen gelesen werden, obwohl (wie Frauen im Allgemeinen) keine von ihnen die eigentliche Quelle angibt. ${ }^{82}$ Frau Liedemann zitiert sogar aus denselben Betrachtungen, die zum 9. August bestellt worden sind, wie Sophia Engelmayer, mit dem Unterschied, dass sie das kleine Gedicht am Anfang des Textes (das eigentliche Motto) ins Album von Fistrovits kopiert hat, während Frau Sophia das sechszeilige Haller-Zitat am Ende der Betrachtung benutzt hat. Fräulein Liedemann hat - im Gegensatz zu den Vorigen - den ersten Band der Ausgabe in die Hände genommen und das ausgewählte Gedicht ähnlich wie ihre Mutter buchstabengetreu wiedergegeben. Anhand dieser Überlegungen halte ich es für sehr wahrscheinlich, dass die ebenso aus Leutschau stammende Sophia Engelmayer den sechszeiligen Auszug, ähnlich wie Johann Michael Schmer, der laut seiner Quellenangabe sicherlich die Ausgabe von Tiede verwendet hat, aus den Unterhaltungen in das ihr angebotene Album kopiert hat. Das heißt, dass das Haller-Zitat von Sophia Engelmayer nicht über die ungarische Rezeption von Haller, sondern vielmehr über jene von Johann Friedrich Tiede - der sich an der Stelle gar nicht auf Haller bezieht - informiert, obwohl die Leutschauer Frau auf gar keine Weise auf den deutschen Prediger hinweist. Bei der Beurteilung des Eintrags von Eleonora Donner müssen wir unbedingt in Betracht ziehen, dass im Ödenburger Autograph die letzte Zeile des Zitats ein wenig anders zu lesen ist als in der Ausgabe von Tiede. ${ }^{83}$ Wir können uns aber auch so ganz

\footnotetext{
${ }^{81}$ IAA, 12,212., 12,213. Die zwei Einträge sind übrigens nebeneinander im Album zu finden, auf den Seiten 91v-92r.

${ }^{82}$ Tiede $1775,2: 83$ und 1:325.

${ }^{83}$ Haller hat übrigens auch selbst den ursprünglichen Text der Verszeile in der Berner Ausgabe von 1777 (worauf ich schon hingewiesen habe) ein wenig modifiziert (,Zum Werkzeug stiller Tugend macht“ - Hervorhebung von mir, M. L.). Anhand seiner Bemerkung zur Textstelle lässt sich feststellen, dass der schweizerische Dichter hier die Version von Christian Fürchtegott Gellert übernommen hat, der Hallers Gedichte oft nachgefeilt zitierte (,Eine Verbesserung vom liebenswürdigen Gellert“). Vgl. Albrecht von Hallers Gedichte, herausgegeben und eingeleitet von Dr. Ludwig HIRZEL (Frauenfeld: Verlag von J. Huber, 1882), 18. Aus der Sicht der Weiteren ist es wichtig zu bemerken, dass die jetzt erwähnte Variante von Gellert im Text der Moralischen Vorlesungen zu lesen ist, die erstmals 1770 erschienen sind. Vgl. Christian Fürchtegott GELLERT, Moralische Vorlesungen, Moralische Charaktere, herausgegeben von Sibylle SPäTH, Gesammelte Schriften VI. (Berlin-New York: De Gruyter, 1992), 176. Es ist ferner interessant, dass die ominöse Verszeile in buchstäblich derselben Form von Eleonora Donner in einem Brief von Gellert (von 1761 aus Leipzig) zu finden ist, und zwar zusammen mit den vorangehenden fünf Zeilen, obwohl deren Text an zwei Stellen bedeutend vom Original Hallers abweicht. Vgl. C. F. Gellerts Briefwechsel, herausgegeben von John F. REYNOLDS, III. 1760-1763 (Berlin-New York: De Gruyter, 1991), 89-90.
} 
sicher sein, dass auch sie aus einer indirekten Quelle zitiert, das heißt, sie zitiert auch nicht „Haller“ selbst.

Die Sache wird übrigens dadurch weiter verkompliziert, dass das (in der derzeitigen Aufarbeitung) früheste Haller-Zitat durch einen ungarischen Einträger genau dieselben sechs Zeilen enthält, von denen wir auch bisher schon gesprochen haben. Im Fall dieses Eintrags ist es aber ganz sicher, dass seine Quelle nicht das Buch von Tiede war. Der erwähnte Eintrag wurde im Album des späteren evangelischen Bischofs Gábor Perlaki bewahrt, der zur Zeit der Entstehung des Autographs, am 31. Januar 1754, ein junger Mann war, der sich auf ein ausländisches Studium vorbereitete. ${ }^{84}$ In den mehr als 20 Einträgen, die in gut einem Monat zwischen dem 1. Januar und dem 8. Februar 1754 in sein Album geschrieben wurden, verabschieden sich seine Pressburger Freunde und Bekannten von ihm. Unter ihnen ist auch der spätere Bürgermeister der Stadt, Karl Gottlieb Windisch. Und Windisch kann die sechs Zeilen seines Eintrags von 1754 aus rein chronologischen Gründen nicht aus Tiedes Buch kopiert haben. ${ }^{85}$ Es scheint natürlich trotzdem sicher zu sein, dass er gerade diese Zeilen nicht unmittelbar aus dem originalen Gedicht von Haller gewählt hat, aber durch welches Medium genau angeregt er diese Passage zitiert hat, ist in diesem Fall schwieriger zu erraten. Es ist aber immerhin soweit sicher, dass vor 1754 außer den schon erwähnten Johann Heinrich Schütte und Friedrich Boerner auch der Herausgeber des schon zuvor erwähnten moralischen Wochenblatts Der Glückselige, Johann Justinus Gebauer, die sechs Zeilen publiziert hat, nämlich in einem 1748er Jahrgang eines anderen moralischen Wochenblatts (ebenfalls aus Halle), Der Gesellige, in dem sie als Motto eines Aufsatzes erscheinen. ${ }^{86}$ Die Quellenangabe unter dem Motto (,Haller“) weist eindeutig darauf hin, dass der zitierte Auszug vom schweizerischen Dichter stammt. Ob Windisch (sagen wir mal) die erwähnte Nummer von Der Gesellige bis 1754 erhielt, wissen wir nicht, ebenso wie wir auch keine Informationen darüber haben, wann er Hallers Dichtung kennengelernt hat. Ich halte es aber für ausgeschlossen, dass ihm der Name und die Dichtung des schweizerischen Dichters zur Zeit seines Eintrags unbekannt waren.

Konkrete Daten haben wir aber erst aus späteren Zeiten. Laut seines Bibliothekskatalogs von 1792 hatte er zum Beispiel sicherlich drei Haller-Bände (und in einem dieser drei waren die Gedichte von Haller zu lesen), die er in der 1774 beginnenden Reihe mit dem Titel Sammlung der besten deutschen prosaischen Schriftsteller und Dichter von Christian Gottlob Schmieder aus Karlsruhe gekauft hat. ${ }^{87}$ Im moralischen Wochenblatt Freund der Tugend, das von Windisch

\footnotetext{
${ }^{84}$ Evangélikus Országos Levéltár [Evangelisches Landesarchiv], VI. 76. Über Perlaki siehe: ZováNYI Jenő, Magyarországi Protestáns Egyháztörténeti Lexikon [Lexikon für die ungarische protestantische Kirchengeschichte], hg. von LADÁNYI Sándor (Budapest: Magyarországi Református Egyház Zsinati Irodájának Sajtóosztálya, 1977), 468.

${ }^{85}$ Der Eintrag von Windisch: IAA, 2,454.

${ }^{86}$ Der Gesellige, 1748. Stück 36. 295-304, 295. Der Autor des Aufsatzes benutzt das Pseudonym „Herr Schriebler Horribilicribrifax gebürtig aus Scaranutz“. Seine kurze Arbeit behandelt das Thema der „Ehre“, zu der das Gedicht von Haller (Über die Ehre), genauer dessen sechs Zeilen, sich selbstverständlich als Motto anbieten. Der Autor zitiert übrigens auch noch einen anderen Teil desselben Gedichts im Text seines Aufsatzes (auf Seite 302).

${ }^{87}$ Jozef TANCER, Im Schatten Wiens: Zur deutschsprachigen Presse und Literatur im Pressburg des 18. Jahrhunderts, Presse und Geschichte - Neue Beiträge 32. (Bremen: Lumière, 2008), 235. In der Schmiederschen Ausgabe der Gedichte Hallers war natürlich auch der gesamte Text des Gedichts Über die Ehre enthalten.
} 
gegründet und editiert wurde, taucht Hallers Name schon 1767 mehrmals auf, in eben jenen Schriften, in denen es auch um Erziehung und die erwünschte Bildung der Frauen geht. ${ }^{88}$ Und zwar ohne jegliche Erklärung, einfach in der Form „Haller“, unter anderem neben den Namen anderer deutscher Dichter (,Utz“, „Gellert“, „Geßner“, „Hagedorn“, „Rabener“, „Zacharia“, „Kleist“, „Kramer“). Das heißt, dass die Kenntnis der Namen dieser Autoren sogar im Kreis der Leser eines Wochenblattes erwartet werden konnte, obgleich diese Ausgaben nicht an die meistgebildeten Intellektuellen gerichtet wurden. ${ }^{89}$ Der autodidaktische Windisch galt trotz fehlenden universitären Abschlusses als wahrer Intellektueller. Zwischen 1764 und 1773 wurde die Preßburger Zeitung von ihm herausgegeben - obwohl sein Name in der Zeitung selbst nicht vorkommt -, der er ab 1767 nacheinander auch drei, zum Teil deutsche moralische Wochenblätter nachahmende Beilagen anschließt, die gleichzeitig die Vorbilder seiner späteren wissenschaftlichen Magazine, des Ungrischen Magazins und des Neuen Ungrischen Magazins waren. Er führte einen umfangreichen Briefwechsel mit ungarischen und ausländischen Wissenschaftlern und in diesen Briefen hat er oft seine Freunde, zum Beispiel Dániel Cornides und János Seivert, über Nachrichten aus der Welt der Wissenschaften oder zu neu erschienenen Büchern informiert. ${ }^{90}$ Er hat wissenschaftliche und populärwissenschaftliche Aufsätze publiziert; einer der Letzteren, ein Geschichtsbuch, wurde im Pressburger evangelischen Lyzeum in der Klasse der Grammatisten und der Donatisten zum Lehrmaterial. ${ }^{91}$ Er war Mitarbeiter der ersten Wiener Wochenschrift Die Welt, die von Christian Gottlob Klemm editiert wurde, ${ }^{92}$ gleichzeitig Mitglied mehrerer (so auch der Augsburger und Olmützer) wissenschaftlicher Gesellschaften und gründete in Pressburg sogar selbst eine Gesellschaft. Deren Mitglieder stammten zunächst (d. h. 1752, somit schon zwei Jahre vor seinem Eintrag) vermutlich aus seinem engeren Freundeskreis, sie wurde aber von 1759 bis 1762 schon als öffentliche Gesellschaft unterhalten, deren Mitglieder unabhängig von ihrer religiösen und nationalen Zugehörigkeit oder sozialen Lage aufgenommen werden konnten. Mit der Dichtkunst beschäftigten sie sich jedenfalls schon ab 1752. ${ }^{93}$ Während der regelmäßigen Lesungen hat am häufigsten, insgesamt 26-mal, Windisch selbst eine seiner Schriften vorgestellt, meistens ein literarisches Werk, zum Beispiel eine Ode

${ }^{88}$ Der Freund der Tugend, 1767. Stück 7 und 15. Zitiert durch: Jozef TANCER, „Die Pressburger Moralischen Wochenschriften im 18. Jahrhundert", in Wynfrid Kriegleder - Andrea SeIDler - Joseph TANCER (Hg.), Deutsche Sprache und Kultur im Raum Pressburg. Presse und Geschichte - Neue Beiträge 4 (Bremen: Lumière, 2002), 207-216, 215; und TANCER, Im Schatten. . ., 161-162.

${ }^{89}$ SEIDLER Andrea, „A nő mint a 18. századi periodikumok olvasója (A folyóirat-hirdetések, az előszavak és az ajánlások forrásértékéről)“ " [Die Frau als Leserin von Periodika im 18. Jahrhundert (Über den Quellenwert von Zeitschriftenannoncen, Vorworten und Widmungen)], Magyar Könyvszemle 115 (1999): 91-97, 92.

${ }^{90}$ SeIDLER Andrea, Briefwechsel des Karl Gottlieb windisch. Magyarországi tudósok levelezése V. [Der Briefwechsel ungarischer Gelehrter V]. (Budapest: Universitas Kiadó, 2008), 12-13.

${ }^{91}$ Markusovszky Sámuel, A pozsonyi ág. hitv. evang. lyceum története kapcsolatban a pozsonyi ág. hitv. evang. egyház múltjával [Geschichte des evangelischen A. B. Lyzeums in Pressburg] (Pozsony: Wigand, 1896), 380.

${ }^{92}$ TANCER, „Die Pressburger...“, 208-209.

${ }^{93}$ Hegedűs Béla, „Über die Pressburgische Gesellschaft der Freunde der Wissenschafften“, in Wynfrid KRIEGLEDER Andrea SeIDler - Joseph Tancer (Hg.), Deutsche Sprache und Kultur im Raum Pressburg. Presse und Geschichte Neue Beiträge 4 (Bremen: Lumière, 2002), 53-64. 
mit dem Titel Die Hunnen oder sein Gedicht Der Tod Abels. ${ }^{94}$ Solche Ambitionen zeigt auch eines seiner Gedichte, das im Album von Pál Fábri zu lesen ist und das während eines dichterischen Wettbewerbs im Jahre 1753 ins Stammbuch geschrieben wurde. ${ }^{95}$

Bezüglich der gleichlautenden Haller-Zitate der Leutschauer und Ödenburger Frauen haben wir gezeigt, dass diese - wegen des indirekten Zitierens - nicht viel mit der ungarischen HallerRezeption zu tun haben. Das auf ähnliche Weise indirekte Haller-Zitat von Windisch zeigt aber, in diesem Fall an dem Beispiel Windischs, dass die Einträger den durch eine sekundäre Quelle beschworenen Autor auch unabhängig davon kennen mögen. Und wie 1754 offenbar auch Windisch die Gedichte von Haller gekannt hat, konnten sie 1779 auch Sophia Engelmayer oder Eleonora Donner bekannt gewesen sein. Im Fall der Zitate, die in den Alben mehrmals oder oft auftauchen, ist also Vorsicht geboten.

Wenn wir die im IAA identifizierten Haller-Zitate in dieser Hinsicht untersuchen, bekommen wir ein ziemlich eigenartiges Ergebnis. In den bisher aufgearbeiteten 53 Haller-Zitaten zitieren die Autoren nämlich lediglich 10 Haller-Gedichte, und zwar oft dieselben Zeilen: ${ }^{96}$

\begin{tabular}{|c|c|c|c|}
\hline Titel des Gedichtes & $\begin{array}{l}\text { Zitierte } \\
\text { Verszeilen }\end{array}$ & Kein Hungarus-Einträger & Hungarus-Einträger \\
\hline \multirow[t]{5}{*}{ Über die Ehre } & $85-96$ & IAA, 7,780 & \\
\hline & 193-194 & IAA, 8,933 & \\
\hline & $193-195$ & IAA, 9,631 & \\
\hline & $193-198$ & IAA, 7,778 & \\
\hline & $217-222$ & & IAA, 2,454, 9,373, 11,218 \\
\hline \multirow[t]{5}{*}{ Die Tugend } & $1-4$ & IAA, 8,687 & \\
\hline & $37-40$ & & $\begin{array}{c}\text { IAA, 8,125, 11,379,* } \\
12,094\end{array}$ \\
\hline & $41-44$ & IAA, 4,233, 9,465, 11,692 & IAA, 5,228, 7,770 \\
\hline & $41-48$ & & IAA, 9,108 \\
\hline & $51-52$ & IAA, 6,166, 7,797 & IAA, 11,698 \\
\hline
\end{tabular}

(continued)

\footnotetext{
${ }^{94}$ SzELEstei N. László, „Tudományok és irodalom Windisch Károly pozsonyi tudós társaságában (1752-1762)“ [Die Wissenschaften und die Literatur in der Pressburger Gelehrtengesellschaft von Károly Windisch (1752-1762)], in Scientiarum miscellanea: Latin nyelvü tudományos irodalom Magyarországon a 15-18. században [Scientiarum miscellanea: Lateinsprachige wissenschaftliche Literatur im Ungarn des 15.-18. Jahrhunderts], hg. von KASZA Péter - KISS FARKAS Gábor - MolNÁr Dávid (Szeged: Lazi, 2017), 219-228, 222-228.

${ }^{95}$ Die Teilnehmer des Wettstreites waren also: Windisch (IAA, 9,061.), Georg Ferdinand Pamer (IAA, 9,062.) und Kovács János (IAA, 9,063.). Vgl. FAjT Anita, „Poetischer Streit in einem Stammbuch: Fábri, Windisch, Pamer und Gottsched“, in Zur kulturellen Funktion von kleiner Differenz: Verwandtschaften, Freundschaften und Feindschaften in Zentraleuropa, hg. von András Balogh F. und Christoph Leitgeb (Wien: Praesens Verlag, 2017), 253-261.

${ }^{96}$ In der Tabelle sind die „Rekordzahlen“ hervorgehoben, die Einträge von Frauen kennzeichnen.
} 


\section{Continued}

\begin{tabular}{|c|c|c|c|}
\hline Titel des Gedichtes & $\begin{array}{l}\text { Zitierte } \\
\text { Verszeilen }\end{array}$ & Kein Hungarus-Einträger & Hungarus-Einträger \\
\hline \multirow[t]{3}{*}{ Über den Ursprung des Übels } & II. $189-190$ & & IAA, 11,530 \\
\hline & III. 81-82 & IAA, 4,792 & IAA, 6,045, 12,219 \\
\hline & III. 82 & IAA, 9,161 & IAA, $7,341,10,000$ \\
\hline \multirow[t]{2}{*}{ Die verdorbenen Sitten } & $221-222$ & IAA, 6,150 & IAA, 3,103, 12,120 \\
\hline & $229-230$ & & IAA, 3,368 \\
\hline \multirow{7}{*}{$\begin{array}{l}\text { Gedanken über Vernunft, } \\
\text { Aberglauben und Unglauben }\end{array}$} & $7-10$ & IAA, 9,227 & \\
\hline & $97-98$ & & IAA, 8,285 \\
\hline & 201-202 & & IAA, 2,862 \\
\hline & 292 & IAA, $7,646,8,308$ & \\
\hline & $335-336$ & IAA, 8,930 & \\
\hline & 372 & IAA, 11,658 & \\
\hline & $387-388$ & & IAA, 9,554 \\
\hline \multirow{5}{*}{$\begin{array}{l}\text { Die Falschheit menschlicher } \\
\text { Tugenden }\end{array}$} & $105-108$ & & IAA, 5,266 \\
\hline & 289 & IAA, 8,879, 8,905, 9,543 & \\
\hline & $289-290$ & & IAA, 8,065 \\
\hline & $322-324$ & IAA, 11,693 & \\
\hline & $365-366^{* *}$ & IAA, 11,704 & \\
\hline \multirow[t]{3}{*}{ Gedanken bei einer Begebenheit } & $1-8$ & IAA, 7,869 & \\
\hline & $3-4$ & IAA, 3,073 & \\
\hline & $13-16$ & & IAA, 8,069 \\
\hline \multirow[t]{2}{*}{ Morgen-Gedanken } & $41-44$ & IAA, 6,171 & \\
\hline & $41-48$ & IAA, 10,964 & \\
\hline $\begin{array}{l}\text { Antwort an Herrn Johann Jakob } \\
\text { Bodmer }\end{array}$ & $125-126$ & IAA, 10,453 & \\
\hline Die Alpen & $1-2 * * *$ & IAA, 8,965 & \\
\hline
\end{tabular}

*Es muss angemerkt werden, dass der Einträger im Fall der Rekorde IAA 8,125 und 11,379 dieselbe Person, József Podmaniczky, ist. Er ist der Einzige, der sogar in mehreren (zwei) Einträgen vom schweizerischen Dichter zitiert.

${ }^{* *}$ Diese Verszeilen kommen im endgültigen Text des Gedichts nicht vor, obwohl sie in der Berner Ausgabe von 1777 als Variante des Autors unter den Bemerkungen auftauchen. Die Laufnummern verweisen hier auf die Berner Ausgabe von 1732.

${ }^{* * *}$ Die angegebenen Laufnummern verweisen auch hier auf die Berner Ausgabe von 1732. Diese zwei Zeilen kommen in der Berner Ausgabe von 1777 unter den Bemerkungen ebenfalls als Variante des Autors vor (auf Seite 304). 
Unserer Tabelle kann entnommen werden, dass die ungarischen Einträger ausschließlich aus denselben Haller-Gedichten zitiert haben wie die deutschen, obwohl ihr Repertoire um drei Gedichte kleiner ist, denn diese drei (unter ihnen das berühmteste Gedicht Hallers) werden überhaupt nicht verwendet (Morgen-Gedanken, Antwort an Herrn Johann Jakob Bodmer, Die Alpen). Es wäre natürlich schwierig zu entscheiden, ob dies ein Zufall ist und wir nach der Bearbeitung von mehr, vielleicht noch viel mehr Haller-Zitaten anders formulieren sollten, aber es ist auch so ersichtlich, dass unsere Einträger nicht ihrem eigenen Geschmack oder eventuellen Lektüren entsprechend aus dem Lebenswerk wählen. Dies wird auch durch die Untersuchungen Katrin Henzels untermauert. Die von ihr gefundenen insgesamt 19 Haller-Zitate stammen nämlich auch aus den Gedichten in unserer Tabelle: Der Zusammenfall ist ganz offensichtlich. ${ }^{97}$ Sie konnte aus zwei bei uns aufgeführten Gedichten (Gedanken bei einer Begebenheit, MorgenGedanken) keine Zitate identifizieren und sie hat ein aus dem im IAA fehlendes (auch im 18. Jahrhundert sehr beliebtes) Gedicht mit dem Titel Doris gefunden, aber davon abgesehen fallen die beiden Listen gänzlich zusammen. Sie zeigen sogar bezüglich der Popularität der einzelnen Gedichte bedeutende Ähnlichkeiten. Bei den Gedichten Antwort an Herrn Johann Jakob Bodmer und Die Alpen gibt es auch bei ihr nur je einen Treffer (wie im IAA) und als das beliebteste Gedicht (mit 7 Treffern) erwies sich Die Tugend (wie auch im IAA, mit 13 Treffern), aber in Bezug auf das Gedicht hat schon Steinhilber von einer ,,abnormen“ Beliebtheit gesprochen. ${ }^{98}$ Sogar hinsichtlich der zitierten Verszeilen ist die Überlappung groß. ${ }^{99}$ Es ist vielleicht nicht so signifikant, dass die erste und die letzte Strophe des Gedichtes (die Zeilen 1-4 und 49-52) auch in dem von Henzel untersuchten Material vorkommen (je einmal), dagegen kommt Strophe 11 (die Zeilen 41-44) in den Leipziger Einträgen viermal vor, wie sie auch im IAA von fünf Personen zitiert wird. Dasselbe gilt für die Zeilen des früher bereits oft erwähnten Über die Ehre: Henzel hat einmal Strophe 33 des Gedichtes als Zitat identifiziert (die Zeilen 193-198), von der 2 Zeilen (die Zeilen 193-194) von Justus Claproth, dem Professor der juristischen Fakultät an der Universität Göttingen (und einem Erneuerer der Papierproduktion), ins Stammbuch von Sándor Podmaniczky eingetragen werden, drei Verszeilen (die Zeilen 193-195) tauchen im Eintrag von Johann Christoph Buch auf (im Album von Sámuel Hajnóczy) und die ganze Strophe wird von Heinrich Wilhelm Mauer in einem weiter oben schon erwähnten Autograph ins Stammbuch von Samuel Coellnberger kopiert. Und natürlich können wir auch wieder die uns schon so bekannten sechs Zeilen antreffen (Strophe 37), die Henzel einmal in dem untersuchten Material finden konnte.

Alles in allem kann festgestellt werden, dass in den im IAA bisher aufgearbeiteten 53 Haller-Zitaten die Einträger insgesamt 34 Textstellen aus zehn Gedichten des gesamten Lebenswerks verwendet haben. Wir können auch noch hinzufügen - und jetzt rechnen wir nicht mit den mangelhaften Daten von Henzel -, dass die Zahl der bloß einmal zitierten Texte derzeit bei 25 liegt, das heißt, dass die Mehrheit unserer Einträger, die Haller zitiert haben, auch von anderen zitierte Zeilen in die ihnen angebotenen Alben kopiert haben. Die Zahl der

\footnotetext{
${ }^{97}$ Henzel, Mehr als..., 360, 455.

${ }^{98}$ Steinhilber, Von der Tugend..., 347. Er hat die Verwendung des Gedichts in 26 Fällen nachgewiesen.

${ }^{99}$ An dieser Stelle möchte ich anmerken, dass den Daten von Henzel leider nicht in jedem Fall zu entnehmen ist, aus welchen Zeilen des bestimmten Gedichtes genau das Zitat stammt. Dies ist der Fall bei den Gedichten Antwort an Herrn Johann Jakob Bodmer, Die Alpen, Die Falschheit menschlicher Tugenden, Gedanken über Vernunft, Aberglauben und Unglauben und Über den Ursprung des Übels. Vgl. Henzel, Mehr als..., 455.
} 
nur zweimal identifizierten Textstellen liegt zurzeit zwar bei lediglich eins, der dreimal zitierten aber bei sieben und es gibt einen Auszug, der sogar fünfmal vorkommt. ${ }^{100}$ Anscheinend war also der in den Alben verwendete Haller-Textkorpus eigentlich ziemlich klein und begrenzt, im Vergleich zum gesamten Lebenswerk des Dichters. Es ist weiterhin offenbar, dass ungarische Einträger dieselben Gedichte und oft dieselben Textstellen benutzt haben wie deutschsprachige. Nach dieser von Männern eingeführten Gewohnheit haben sich auch die Frauen gerichtet. Ihre Zitate stammen in jedem Fall aus Gedichten, die auch von anderen (von Männern) zitiert wurden; sechs von neun zitieren sogar Verszeilen, die von anderen (also von Männern) benutzt wurden.

Wenn wir genau die Grenzen des in den Alben regelmäßig benutzten Haller-Korpus kennen würden, könnten wir die Einträge der Haller zitierenden „Außenseiter“ erkennen: Vermutlich solche Einträge, in denen die Einträger von anderen ignorierte Gedichte oder Textstellen zitieren. Wir bewegen uns in diesem Fall zurzeit natürlich nicht auf sicherem Boden, da sich von diesen ,ignorierten“ Textstellen während der weiteren Datensichtung sogar herausstellen könnte, dass sie in Wirklichkeit oft zitierte Teile sind. Vielleicht ist es aber trotzdem nicht uninteressant, zumindest einige solche Autographe zu untersuchen. Zum Beispiel die Einträge jener drei Frauen, die von anderen (bei der aktuellen Aufarbeitung) nie zitierte Textstellen zitieren.

\section{AMALIA TRANGUS UND DIE GEDANKEN BEI EINER BEGEBENHEIT}

Einer dieser Einträge wurde früher schon erwähnt, nämlich die Inscriptio von Amalia Trangus aus Zipser Neudorf, die aus Gedanken bei einer Begebenheit die Zeilen 13-16 zitiert hat:

Nein, bettle wer da will des Glückes eitle Gaben,

Im Wunsche gross, klein im Genuss;

Von mir soll das Geschick nur diese Bitte haben,

Gleich fern von Noth und Uiberfluss. ${ }^{101}$

Henzel - wie wir auch schon gesehen haben - konnte die Verwendung des Gedichts in den Leipziger Einträgen nicht nachweisen. Im IAA wird es aber sowohl in einem Tübinger als auch in einem Jenaer Autograph zitiert, beide Male die ersten Zeilen. ${ }^{102}$ Die letzte Strophe konnte aber bisher nur im Eintrag von Fräulein Trangus identifiziert werden. Das als Teil von Versuch Schweizerischer Gedichte erstmals 1748 (d. h. in der vierten Ausgabe) publizierte Gedicht war

\footnotetext{
${ }^{100}$ Ich habe hier zwei zitierte Textstellen nur in dem Fall als identisch betrachtet, wenn diese eine vollkommene Übereinstimmung gezeigt haben. Die Zeilen 193-194 aus Über die Ehre sind jetzt auf keinen Fall identisch mit den Zeilen 193-195 aus Über die Ehre. Wenn ich auch die „Teilidentitäten“ miteinberechnet hätte, wäre die Zahl der nur einmal zitierten Textstellen natürlich noch kleiner (18 statt 25). Wenn ich auch die Daten von Henzel verwenden würde, die in dieser Hinsicht auswertbar sind, wäre sie noch kleiner. Im Zusammenhang damit würde die Zahl der mehrmals zitierten Textstellen natürlich wachsen.

${ }^{101}$ IAA, 8,069.

${ }^{102}$ Christian Karl Philipp Vollmar die Zeilen 1-8 (IAA, 7,869), Johann Christian Christoph Rüdiger die Zeilen 3-4 (IAA, 3,073).
} 
übrigens beliebt, was dadurch ersichtlich wird, dass im 18. Jahrhundert mehrere Umschriften davon entstanden sind. Eine davon ist die mehrmals publizierte Variante von Karl Wilhelm Ramler, die auch einen neuen Titel trägt. Im zweiten Band des Lehrbuchs von Christian Gottfried Schütz von 1778 können wir zum Beispiel auch den Ramlerschen Text finden, ${ }^{103}$ wie auch im Lesebuch von Aegidius Jais, das zuerst 1784 herausgegeben wurde und ebenfalls als Schulbuch gedacht war. ${ }^{104}$ Ramler selbst hat natürlich auch mehrmals das Gedicht publiziert (z. B. 1774, also noch zu Lebzeiten Hallers), dessen letzte Strophe er lediglich an einer Stelle geändert hat. ${ }^{105}$ Als Ergebnis der winzigen, aber scheinbar doch nicht unbedeutenden Modifikation wurde aus dem originalen „Von mir“ der dritten Zeile in der Strophe ein „Von uns“, was übrigens ganz mit der Grundtendenz der Umdichtung von Ramler übereinstimmt. Die andere Version stammt von der 1753 zur Dichterin gekrönten Johanna Charlotte Unzer, die offensichtlich aus der Umschrift von Ramler gearbeitet hat. Den Titel des Gedichts hat auch sie geändert (Ermunterung zur Fröhlichkeit) und außerdem noch zahlreiche Veränderungen an dem schon von Ramler weitergeschriebenen Text vorgenommen. Zum Beispiel sieht die von Fräulein Trangus zitierte letzte Strophe in ihrer Version folgendermaßen aus:

Nein! Bettle, wer da will, des Glückes eitle Gaben,

Im Wunsche gross, klein im Genuss:

Nur diese Bitte soll von mir das Schicksal haben:

Gleich fern von Noth und Überfluss! ${ }^{106}$

Die Strophe selbst - besonders ihre originale Version - scheint trotzdem nicht selbständig, vom Gedicht getrennt in den gedruckten Medien ,verstreut“ zu sein, wo wir höchstens ihre permutierten Versionen antreffen können und auch diese nur in einigen Fällen. Zum Beispiel in dem zweibändigen ,gesellschaftlichen Spiel-Buch“, das 1773 in Quedlinburg und in Blankenburg herausgegeben wurde, unter den Begrüßungen (,Gesundheiten“) und hinsichtlich der ersten Zeile auch hier mit einem umgeschriebenen Text (,Es bettle wer da will des Glückes eitle Gaben"). ${ }^{107}$ Dieselbe Umschrift ist aber auch im Kalender mit dem Titel Oesterreichischer Toleranz-Bote zu lesen, der 1786 in Wien erschienen ist. ${ }^{108}$ Diese Ausgaben, die im Gegensatz zu Amalia Trangus den Namen Hallers nicht einmal erwähnen, konnten also auf keinen Fall als Quelle für das Fräulein aus Zipser Neudorf dienen. Es ist deswegen denkbar, dass der Eintrag von Amalia Trangus, der den originalen Text der Strophe zitiert, wirklich als Eintrag eines Außenseiters zu interpretieren ist; dies im Gegensatz - zum Beispiel - zu den Autographen derer, die die Zeilen 41-44 aus Die Tugend zitiert haben. Dies kann theoretisch auf ein

${ }^{103}$ Christian Gottfried SchüTZ, Lehrbuch zur Bildung des Verstandes und des Geschmacks: Zum Behufe des öffentlichen Schul-und Privatunterrichts. I-II. (Halle: Curt, 1776-1778), 2:247-248.

${ }^{104}$ Aegidius JAIS, Lesebuch für meine Schüler zur Bildung ihres Herzens (Salzburg: Mayr, 1784), 140-141.

${ }^{105}$ Karl Wilhelm RAmLER, Lyrische Bluhmenlese (Leipzig: Weidmann und Reich, 1774), 229. Der neue Titel: Aufmunterung zur Vergnügsamkeit.

${ }^{106}$ Friedrich von MatThisson, Lyrische Anthologie. Band 19 (Zürich: Orell Füssli, 1807), 173.

${ }^{107}$ In: Zeitverkürzende Winterlustbarkeiten für junge Leute in Gesellschaften II. (Bestehend aus Gesundheiten, Räthseln, Kunststücken, Erzehlungen u.a.m.) (Blankenburg: Reußner, 1773), 95.

${ }^{108}$ Herbstmonat, Sitten-und Klugheitslehren. 
persönlicheres, wenn wir so wollen, ,intimeres“ Verhältnis zur Dichtung Hallers verweisen. Im (536 Seiten langen) einführenden Aufsatz der kritischen Ausgabe von Ludwig Hirzel spricht dieser von den Briefen von Luise Adelgunde Victorie Kulmus an ihre Freundinnen, in denen sie Haller als ,mein[en] Lieblingsdichter“ erwähnt. Diese gebildete und kluge Frau - die Frau von Gottsched und selbst Schriftstellerin - zitiert in ihren Briefen oft die Gedichte von Haller, offenbar anhand ihrer intensiven Lektüren. Einmal gerade die letzte Strophe aus Gedanken bei einer Begebenheit, die auch Fräulein Trangus zitiert. ${ }^{109}$ Ich behaupte natürlich nicht, dass das Verhältnis von Fräulein Trangus zu den Gedichten von Haller gänzlich zu dem von Frau Kulmus ähnlich gewesen sei - schon aber, wenn auch bedingt, dass ihr Eintrag ein Abdruck eines persönlichen (wenn auch von der Mode geleiteten) Erlebnisses mit dem zitierten Gedicht ist. Damit scheint übereinzustimmen, dass das Fräulein aus Zipser Neudorf auch eine Quelle zu den zitierten Verszeilen angibt. Wenn wir von den Einträgen der Frauen absehen, die die Bibel zitieren und sich so darauf beziehen, können wir dasselbe nur von 6 Prozent der Einträger unter den ungarischen Frauen im 18. Jahrhundert feststellen.

(Fortsetzung folgt)

Open Access. This is an open-access article distributed under the terms of the Creative Commons Attribution 4.0 International License (https://creativecommons.org/licenses/by/4.0/), which permits unrestricted use, distribution, and reproduction in any medium, provided the original author and source are credited, a link to the CC License is provided, and changes - if any - are indicated. (SID_1)

${ }^{109}$ Hirzel, Albrecht von Hallers. .., CCCLIX-CCCLX. 